\title{
Breathers in oscillator chains with Hertzian interactions
}

\author{
Guillaume James ${ }^{\mathrm{a}, 1}$, Panayotis G. Kevrekidis ${ }^{\mathrm{b}}$, Jesús Cuevas ${ }^{\mathrm{c}}$. \\ ${ }^{a}$ Laboratoire Jean Kuntzmann, Université de Grenoble and CNRS, BP 53, 38041 \\ Grenoble Cedex 9, France. \\ ${ }^{\mathrm{b}}$ Department of Mathematics and Statistics, University of Massachusetts, \\ Amherst, Massachussets 01003-4515, USA. \\ ${ }^{\mathrm{c}}$ Grupo de Física No Lineal. Departamento de Física Aplicada I. Escuela \\ Politécnica Superior. Universidad de Sevilla. C/ Virgen de África, 7. 41011 \\ Sevilla, Spain.
}

\begin{abstract}
We prove nonexistence of breathers (spatially localized and time-periodic oscillations) for a class of Fermi-Pasta-Ulam lattices representing an uncompressed chain of beads interacting via Hertz's contact forces. We then consider the setting in which an additional on-site potential is present, motivated by the Newton's cradle under the effect of gravity. Using both direct numerical computations and a simplified asymptotic model of the oscillator chain, the so-called discrete $p$-Schrödinger (DpS) equation, we show the existence of discrete breathers and study their spectral properties and mobility. Due to the fully nonlinear character of Hertzian interactions, breathers are found to be much more localized than in classical nonlinear lattices and their motion occurs with less dispersion. In addition, we study numerically the excitation of a traveling breather after an impact at one end of a semi-infinite chain. This case is well described by the DpS equation when local oscillations are faster than binary collisions, a situation occuring e.g. in chains of stiff cantilevers decorated by spherical beads. When a hard anharmonic part is added to the local potential, a new type of traveling breather emerges, showing spontaneous direction-reversing in a spatially homogeneous system. Finally, the interaction of a moving breather with a point defect is also considered in the cradle system. Almost total breather reflections are observed at sufficiently high defect sizes, suggesting potential applications of such systems as shock wave reflectors.
\end{abstract}

Key words: Hamiltonian lattice, Hertzian contact, discrete breathers, direction-reversing waves, discrete $p$-Schrödinger equation, cantilever arrays.

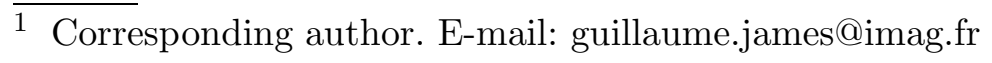

Preprint submitted to Physica D

17 June 2018 


\section{Introduction}

The study of nonlinear waves in granular crystals is the object of intensive research, both from a theoretical perspective and for practical purposes, e.g. for the design of shock absorbers [33,64,26], acoustic lenses [68] or diodes [6]. Due to the nonlinear interactions between grains, several interesting types of localized waves can be generated in chains of beads in contact. Solitary waves are the most studied type of excitations and can be easily generated by an impact at one end of a chain $[58,21,32,51,1,60,64]$. These solitary waves, in the absence of an original compression in the chain (the so-called precompression), differ from classical ones (i.e. KdV-type solitary waves [27]) due to the fully nonlinear character of Hertzian contact interactions. Indeed, their decay is super-exponential and their width remains unchanged with amplitude $[20,69]$.

Another interesting class of excitations consists of time-periodic and spatially localized oscillations. Such waves may correspond to Anderson modes [34] in the presence of spatial disorder, or to defect modes localized at an impurity in a granular chain under precompression [70]. A different class of spatially localized oscillations that occur in the absence of defects consists of discrete breathers, which originate from the combined effects of nonlinearity and spatial discreteness (see the reviews [23,24]). These waves exist in diatomic granular chains under precompression $[7,71,38]$, with their frequency lying between the acoustic and optic phonon bands and can be generated e.g. through modulational instabilities. However, because precompression suppresses the fully nonlinear character of Hertzian interactions, these excitations inherit the usual properties of discrete breathers, i.e. their spatial decay is exponential and their width diverges at vanishing amplitude, i.e. for frequencies close to the bottom of the optic band [38].

For granular systems without precompression, the above discussion raises the question of existence of spatially localized oscillations. Defect modes induced by a mass impurity have been numerically observed on short transients in unloaded granular chains $[31,42]$, but the existence of permanent localized oscillations remains an open question. In this paper, we give a partial answer to this problem by showing the nonexistence of time-periodic spatially localized oscillations in uncompressed granular chains. This result seems surprising at a first glance, because Hertzian models of granular chains fall within the class of Fermi-Pasta-Ulam (FPU) lattices, which sustain discrete breathers under some general assumptions on the interaction potentials and particle masses (see [59] and references therein). However these conditions do not hold for uncompressed granular chains. Using a simple averaging argument, we show that the non-attracting character of Hertzian interactions between grains (repulsive under contact, and vanishing in the absence of contact) precludes the existence of time-periodic localized oscillations, both for spatially homogeneous systems 
and for inhomogeneous chains.

However, in contrast to the above picture, the existence of discrete breathers in a chain of linear oscillators coupled by Hertzian potentials has been recently reported [41]. This model with an on-site potential describes e.g. the small amplitude waves in a Newton's cradle [35], which consists of a chain of beads attached to pendula (see figure 1, left). In [41], static and moving breathers were numerically observed as a result of modulational instabilities of periodic traveling waves, and extremely stable static breathers were generated from specific initial conditions. In addition, a reduced model, the so-called discrete $p$-Schrödinger (DpS) equation was derived as an asymptotic model for small amplitude oscillations in the Newton's cradle, and successfully reproduced the above localization phenomena. The discrete breathers possess special properties both in the original cradle model and the simplified DpS system, i.e. their spatial decay is super-exponential and their width remains nearly constant at small amplitude.

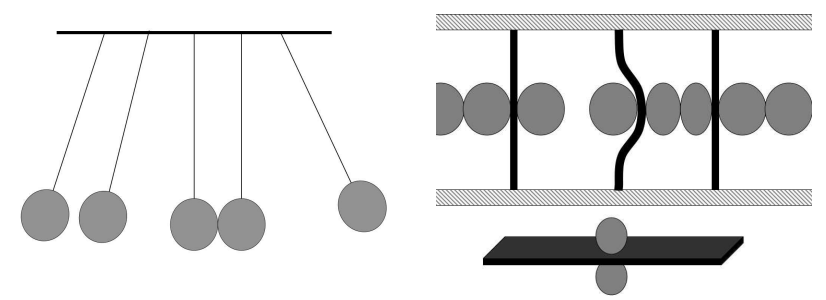

Fig. 1. Left : prototypical Newton's cradle. Right : array of clamped cantilevers decorated by spherical beads (displacements are amplified for clarity).

In this paper we extend the above results in three directions. The first one concerns traveling breather excitations, i.e. localized waves displaying an internal oscillation in addition to their translational motion. We show numerically that such waves can be excited very simply in the cradle system by an impact at one end of a semi-infinite chain, and check that this dynamics is also reproduced by the DpS model. We also discuss some unusual properties of the moving breathers obtained in this way. Due to the fully nonlinear Hertzian interactions, these breathers display a strong localization (super-exponential decay) and their dispersion remains very weak during propagation. In contrast, introducing a precompression attenuates spatial localization and enhances dispersive effects (due to the fact that precompression adds effectively a linear component to Hertzian interactions). We illustrate this idea using both numerical simulations and the discrete nonlinear Schrödinger equation, which allows us to approximate small amplitude traveling breathers under the effect of precompression. In addition, we check that the whole phenomenology remains valid at small amplitude when the linear local potential is replaced by a smooth anharmonic potential. However, such local nonlinearities yield additional phenomena, such as the excitation of a surface mode after the impact for soft local potentials, and for hard potentials the occurence of a direction- 
reversing traveling breather. The latter is reminiscent of excitations known as "boomerons" (direction-reversing solitons) that were found up to now only in particular integrable models (see [16] and references therein).

Our second contribution concerns the computation of static breathers in the Newton's cradle and their numerical continuation. Using a modified GaussNewton method introduced in [10], we obtain branches of site- and bondcentered breathers parametrized by their frequency $\omega>\omega_{0}$ ( $\omega_{0}$ being the frequency of the local linear oscillators). These branches bifurcate from the trivial equilibrium when $\omega \rightarrow \omega_{0}$ and can be continued up to a strongly nonlinear regime. Moreover, the Floquet spectra of these breathers display (in addition to the usual double eigenvalue +1 ) an extra pair of eigenvalues very close to unity. As an effect of this near-degeneracy, small perturbations of the breathers along an associated pinning mode generate a translational motion with negligible radiation, according to the process analyzed in [2]. This provides a connection between these standing breathers and the traveling ones mentioned above. In addition to these numerical computations, we obtain an analytical quasi-continuum approximation of the breather profiles valid at small amplitude. These approximate breathers have a compact support, which provides a reasonable approximation to the super-exponential decay of the exact breathers. This situation is analogous to what is known for the approximation of solitons in uncompressed granular chains $[58,20]$.

Lastly, we examine possible experimental realizations of these kinds of granular lattices and the related observation of moving breathers after an impact, as well as their potential practical usefulness (e.g., in the form of granular protectors; see below). In the usual Newton's cradle, the period of local oscillations (of the order of a second) is much larger than the collision time between two beads (typically of the order of $0.1 \mathrm{~ms}$ [49]), so the propagation of compression pulses and the oscillatory dynamics occur on two well-separated time scales. Moreover, as shown in the present paper, the $\mathrm{DpS}$ regime giving rise to discrete breathers is realized when local oscillations are faster than binary collisions. As a consequence, moving breathers would not be observable in practice in a Newton's cradle. However, we argue that reasonably simple mechanical systems could be tailored so that the oscillation and collision time scales become similar and the DpS regime takes place. As a prototype for which this situation occurs, we consider the chain of identical clamped cantilevers represented in figure 1. Each cantilever is decorated by two spherical beads attached to its center, and the beads of two successive cantilevers are tangent at the ground state. Using a reduced oscillator chain model of this system (calibrated for realistic material parameter values), we check that an impact on the first cantilever generates a moving breather. In addition, we argue that such devices may have potential applications as granular protectors. Indeed, we observe that moving breathers can be almost totally reflected by a localized impurity. 
The outline of the paper is as follows. In section 2 we prove the non-existence of time-periodic spatially localized oscillations in a broad class of Fermi-PastaUlam lattices with non-attracting interactions, including uncompressed granular chains. Section 3 studies the properties of static and moving breathers in granular chains with on-site potentials and in the DpS equation, and highlights the relation between the two models. The new effects introduced by local anharmonic potentials are also discussed. In addition we introduce the mixed granular-cantilever chain, and analyze an impact problem in this system in connection with the previous findings. Section 4 illustrates how precompression modifies the properties of localized waves. Lastly, section 5 concludes this study and analyzes the results from a more general perspective, in connection with possible experiments.

\section{Non-existence of breathers in FPU chains with repulsive inter- actions}

We consider an infinite chain of particles of masses $m_{n}>0$, interacting with their nearest neighbors via anharmonic potentials $V_{n}$. This type of system (which can be thought of in general, i.e., for unequal masses $m_{n}$, as a spatially inhomogeneous FPU lattice) corresponds to the Hamiltonian

$$
\mathcal{H}=\sum_{n \in \mathbb{Z}} \frac{m_{n}}{2} \dot{x}_{n}^{2}+V_{n}\left(x_{n+1}-x_{n}\right)
$$

where $x_{n}$ denotes the particle displacements from the ground state. We consider interaction potentials $V_{n}$ of the form

$$
V_{n}(x)=W_{n}\left[(-x)_{+}\right]
$$

where $(a)_{+}=\max (\mathrm{a}, 0), W_{n} \in C^{1}\left(\mathbb{R}^{+}, \mathbb{R}^{+}\right), W_{n}^{\prime}(0)=0$ and $W_{n}^{\prime}(x)>0$ for all $x>0$. The form of $V_{n}$ implies that particle interactions are repulsive under compression (i.e. for $x<0$ ) and unilateral (interaction forces vanish under extension, i.e. for $x>0$ ).

Moreover we assume

$$
W_{n}^{\prime}(x) \leq f(x) \quad \forall x \in[0, r], \forall n \geq n_{0}
$$

for some real constant $r>0$, integer $n_{0}$ and a monotone increasing function $f \in C^{0}([0, r])$ satisfying $f(0)=0$. For example, these assumptions are satisfied with $f(x)=\sup _{n>n_{0}} W_{n}^{\prime}(x)$ if the functions $W_{n}$ are convex in [0,r] and belong to some finite set for $n \geq n_{0}$ (this is the case in particular for spatially periodic 
systems). Another example is given by Hertzian interactions

$$
W_{n}(x)=\frac{1}{\alpha_{n}+1} \gamma_{n} x^{\alpha_{n}+1}
$$

where the coefficients $\gamma_{n}, \alpha_{n}>0$ depend on material properties and particle geometry. In that case one can choose $f(x)=\gamma x^{\alpha}$ (and $r=1$ ) provided $\gamma_{n} \leq \gamma$ and $\alpha_{n} \geq \alpha>0$ for all $n \geq n_{0}$.

The Hamiltonian (1) leads to the equations of motion

$$
m_{n} \ddot{x}_{n}=V_{n}^{\prime}\left(x_{n+1}-x_{n}\right)-V_{n-1}^{\prime}\left(x_{n}-x_{n-1}\right), \quad n \in \mathbb{Z} .
$$

In what follows we show that under the above assumptions, the only timeperiodic breather solutions of (3) are trivial equilibria. Due to the translational invariance of (1), breathers are defined as time-periodic solutions which converge (uniformly in time) towards translations $x_{n}=c_{ \pm} \in \mathbb{R}$ as $n \rightarrow \pm \infty$. This implies that relative particle displacements vanish at infinity, i.e. one has

$$
\lim _{n \rightarrow \pm \infty}\left\|x_{n}-x_{n-1}\right\|_{L^{\infty}(0, T)}=0
$$

for a $T$-periodic breather. In what follows, we prove in fact a more general nonexistence result of nontrivial periodic solutions vanishing as $n \rightarrow+\infty$.

Theorem 1 All time-periodic solutions of (3) satisfying

$$
\lim _{n \rightarrow+\infty}\left\|x_{n}-x_{n-1}\right\|_{L^{\infty}}=0
$$

are independent of $t$ and increasing with respect to $n$.

Proof. Let us consider a $T$-periodic solution of (3) and integrate (3) over one period. This yields the equality

$$
\bar{F}_{n}=\bar{F}_{n+1}
$$

where $\bar{F}_{n}=\frac{1}{T} \int_{0}^{T} V_{n-1}^{\prime}\left(x_{n}(t)-x_{n-1}(t)\right) d t$ is the average interaction force between masses $n-1$ and $n$. Consequently $\bar{F}_{n}=\bar{F}$ is independent of $n$.

Now let us check that $\bar{F}$ vanishes thanks to the bound (2) uniform in $n$. We have for all $n$

$$
\begin{aligned}
|\bar{F}| & =\frac{1}{T} \int_{0}^{T} W_{n-1}^{\prime}\left[\left(x_{n-1}(t)-x_{n}(t)\right)_{+}\right] d t \\
& \leq\left\|W_{n-1}^{\prime}\left[\left(x_{n-1}-x_{n}\right)_{+}\right]\right\|_{L^{\infty}} .
\end{aligned}
$$


Taking into account (5) and (2), the above inequality yields for $n$ large enough

$$
|\bar{F}| \leq\left\|f\left[\left(x_{n-1}-x_{n}\right)_{+}\right]\right\|_{L^{\infty}}=f\left[\left\|\left(x_{n-1}-x_{n}\right)_{+}\right\|_{L^{\infty}}\right]
$$

since $f$ is increasing. It follows that

$$
|\bar{F}| \leq f\left(\left\|x_{n-1}-x_{n}\right\|_{L^{\infty}}\right) \rightarrow 0 \text { as } n \rightarrow+\infty
$$

hence $\bar{F}=0$.

Now we use the fact that the interactions between particles are repulsive, i.e. we have $-V_{n}^{\prime}(x)=W_{n}^{\prime}\left[(-x)_{+}\right] \geq 0$. Since the $T$-periodic functions $F_{n}(t)=$ $V_{n-1}^{\prime}\left(x_{n}(t)-x_{n-1}(t)\right)$ are negative, continuous and satisfy $\int_{0}^{T} F_{n}(t) d t=0$ as shown previously, we have consequently $F_{n}(t)=0$ for all $t$ and $n$. Using (3), this implies $\ddot{x}_{n}=0$ and thus $x_{n}$ is an equilibrium solution (due to timeperiodicity). Moreover one has $x_{n} \geq x_{n-1}$ since $F_{n}=0$.

We note that the above arguments do not work if an on-site potential is added to (1), because the average interaction forces are no more independent of $n$. In the next section, we numerically show the existence of breathers for such type of nonlinear lattices.

\section{Granular chains with local potentials and their correspondence to the DpS Equation}

We consider a nonlinear lattice with the Hamiltonian

$$
\mathcal{H}=\sum_{n} \frac{1}{2} \dot{y}_{n}^{2}+W\left(y_{n}\right)+V\left(y_{n+1}-y_{n}\right),
$$

where

$$
V(r)=\frac{2}{5}(-r)_{+}^{5 / 2}
$$

The system (6) corresponds to a chain of identical particles in the local potential $W$, coupled by the classical Hertz potential $V$ describing contacts between smooth non-conforming surfaces. Unless explicitly stated, the on-site potential $W$ will be chosen harmonic with

$$
W(y)=\frac{1}{2} y^{2} .
$$

In that case, the dynamical equations read

$$
\ddot{y}_{n}+y_{n}=\left(y_{n-1}-y_{n}\right)_{+}^{3 / 2}-\left(y_{n}-y_{n+1}\right)_{+}^{3 / 2} .
$$

Figure 1 depicts two examples of such systems. In practical situations, the assumption of a local harmonic potential implies that the model will be valid 
for small amplitude waves and suitable time scales on which higher order terms can be neglected. In order to capture higher order effects, different parts of our analysis will be extended to symmetric anharmonic local potentials

$$
W(y)=\frac{1}{2} y^{2}+\frac{s}{4} y^{4}
$$

where the parameter $s$ measures the degree of anharmonicity.

In the work [41], long-lived static and traveling breather solutions of (9) have been numerically observed, starting from suitably chosen localized initial conditions, or from small perturbations of unstable periodic traveling waves. However, the classical result of MacKay and Aubry [50] proving the existence of static breathers near the anti-continuum limit does not apply in that case. Indeed, if Hertzian interactions forces are cancelled (or equivalently, if one considers breathers in the limit of vanishing amplitude), one obtains an infinite lattice of identical linear oscillators, and the nonresonance assumption of reference [50] is not satisfied. Moreover, other existence proofs based on spatial dynamics and the center manifold theorem [40] do not apply, due to the fully-nonlinear character of interaction forces (the same remark holds true in the case of traveling breathers $[36,39,66])$. Variational tools $[3,59]$ may be suitable to obtain existence proofs in this context, but this question is outside the scope of the present paper, where we shall resort chiefly to numerical and asymptotic methods.

In section 3.1, we recall the relation between (9) and the DpS equation derived in [41]. In section 3.2, we numerically compute breather solutions of (9) by the Newton method, and compare them to a quasi-continuum approximation deduced from the DpS equation. Section 3.3 concerns the stability and mobility of breathers in model (6)-(10) and the DpS equation, and the generation of traveling breathers by an impact is studied in the same models in section 3.4. In section 3.5, we consider a general class of granular chains with local potentials, and show that the DpS regime occurs in the above impact problem when local oscillations are faster than binary collisions. Section 3.6 provides an application of the above results to a chain of stiff cantilevers decorated by spherical beads.

\subsection{The discrete $p$-Schrödinger equation}

Small amplitude solutions of system (6)-(8) can be well approximated by an equation of the nonlinear Schrödinger type, namely the discrete $p$-Schrödinger (DpS) equation with $p=5 / 2$

$$
i \dot{v}_{n}=\left(v_{n+1}-v_{n}\right)\left|v_{n+1}-v_{n}\right|^{p-2}-\left(v_{n}-v_{n-1}\right)\left|v_{n}-v_{n-1}\right|^{p-2} .
$$


The most standard model reminiscent of this family of equations is the socalled discrete nonlinear Schrödinger (DNLS) equation, studied in detail in a number of different contexts, including nonlinear optics and atomic physics over the past decade $[45,19]$. However, the DpS equation is fundamentally different in that it contains a fully nonlinear inter-site coupling term, corresponding to a discrete $p$-Laplacian.

To make the connection with the DpS equation more precise, we sum up some basic elements of the analysis of [41]. Let us consider the lattice model (9) and the $\mathrm{DpS}$ equation

$$
2 i \tau_{0} \dot{A_{n}}=\left(A_{n+1}-A_{n}\right)\left|A_{n+1}-A_{n}\right|^{1 / 2}-\left(A_{n}-A_{n-1}\right)\left|A_{n}-A_{n-1}\right|^{1 / 2},
$$

where the time constant $\tau_{0}$ reads

$$
\tau_{0}=\frac{5\left(\Gamma\left(\frac{1}{4}\right)\right)^{2}}{24 \sqrt{\pi}} \approx 1.545
$$

and $\Gamma$ denotes Euler's Gamma function. Given a solution of (12) and $\epsilon>0$ small enough, one obtains an approximate solution of (9)

$$
y_{n}^{\mathrm{app}}(t)=2 \epsilon \operatorname{Re}\left[A_{n}\left(\epsilon^{1 / 2} t\right) e^{i t}\right] .
$$

The approximate solution (13) and amplitude equation (12) have been derived in [41] using a multiple-scale expansion. According to [18], for initial conditions of the form $y_{n}(0)=2 \epsilon \operatorname{Re}\left[A_{n}(0)\right]+O\left(\epsilon^{3 / 2}\right), \dot{y}_{n}(0)=-2 \epsilon \operatorname{Im}\left[A_{n}(0)\right]+O\left(\epsilon^{3 / 2}\right)$ with $\epsilon \approx 0$, this approximation is $O\left(\epsilon^{3 / 2}\right)$-close to the exact solution of (9) at least up to times $t=O\left(\epsilon^{-1 / 2}\right.$ ) (see also numerical results of [41] comparing the DpS approximation and exact solutions of (9)). Moreover, for some family of periodic traveling wave solutions of the DpS equation, the ansatz (13) is $O\left(\epsilon^{3 / 2}\right)$-close to exact small amplitude periodic traveling waves of (9) [41].

Lastly, it is interesting to mention that the DpS equation depends on the terms of (9) up to order $O\left(|y|^{3 / 2}\right.$ ) (see [41], section 2.1). It follows that this equation remains unchanged for smooth anharmonic on-site potentials $W(y)=$ $\frac{1}{2} y^{2}+O\left(|y|^{3}\right)$, because the associated extra nonlinearity is at least quadratic. Consequently, the addition of a local anharmonicity doesn't change the dynamics of (9) for small amplitude waves, on the timescales governed by the DpS equation.

\subsection{Computation of static breathers}

The work of [41] illustrated the existence of time-periodic and spatially localized solutions of the DpS equation. Figures 2 and 3 (top panels) display the 
profiles of spatially antisymmetric or symmetric breather solutions of the DpS equation (11). These are sought by using the standard stationary ansatz for DNLS type equations of the form $v_{n}=\exp (i \mu t) u_{n}$ with $\mu>0$ and $u_{n} \in \mathbb{R}$. The resulting coupled nonlinear algebraic equations read

$$
-\mu u_{n}=\left(u_{n+1}-u_{n}\right)\left|u_{n+1}-u_{n}\right|^{1 / 2}-\left(u_{n}-u_{n-1}\right)\left|u_{n}-u_{n-1}\right|^{1 / 2}
$$

and are solved via a fixed point iteration of the Newton-Raphson type, for free end boundary conditions.

Note that equation (11) has a scale invariance, since any solution $v_{n}(t)$ generates a one-parameter family of solutions $a v_{n}\left(|a|^{1 / 2} t\right), a \in \mathbb{R}$. Thanks to this scale invariance, the whole families of antisymmetric and symmetric breathers can be reconstructed from the case $\mu=1$ of (14). In particular, breather amplitudes are $\propto \mu^{2}$ and the breather width remains unchanged when $\mu \rightarrow 0$, a property that strongly differs from the broadening of DNLS breathers at small amplitude (see e.g. [12], section 3).

In what follows we approach the two breather profiles using a quasi-continuum approximation. Fixing $\mu=1$ and introducing

$$
w_{n}=\left(u_{n+1}-u_{n}\right)\left|u_{n+1}-u_{n}\right|^{1 / 2}
$$

equation (14) becomes

$$
w_{n+1}-2 w_{n}+w_{n-1}+w_{n}\left|w_{n}\right|^{-1 / 3}=0,
$$

where the nonlinear coupling has been linearized (at the expense of having an on-site nonlinearity non-differentiable at the origin). The spatial profiles of figures 2 and 3 suggest to use the so-called staggering transformation $w_{n}=$ $(-1)^{n} f(n)$, which yields

$$
f(n+1)-2 f(n)+f(n-1)=-4 f(n)+f(n)|f(n)|^{-1 / 3} .
$$

Now we look for an approximate solution $F$ of (16). For this purpose we use the formal approximation $F(n \pm 1) \approx F(n) \pm F^{\prime}(n)+\frac{1}{2} F^{\prime \prime}(n)$, in same the spirit as the approximations of soliton profiles performed in reference [58] (the accuracy of this approximation will be checked a posteriori by numerical computations) 2 . This leads to the differential equation

$$
F^{\prime \prime}=-4 F+F|F|^{-1 / 3}
$$

$\overline{2}$ Note that $w_{n}$ corresponds to a spatially modulated binary oscillation, and a continuum approximation is obtained for its envelope, whereas the continuum approximation of [58] was performed on the full soliton profiles. 
which possesses a family of compactly supported solutions $F(x)= \pm g(x+\phi)$, where

$$
g(x)=\left(\frac{3}{10}\right)^{3} \cos ^{6}\left(\frac{x}{3}\right) \text { for }|x| \leq \frac{3 \pi}{2}, \quad g=0 \text { elsewhere. }
$$

Replacing $f$ by its approximation $F$ and performing appropriate choices of sign and spatial shifts in $F$, one obtains the symmetric approximate solutions of $(15)$

$$
w_{n}^{(1)}=(-1)^{n+1} g(n), \quad w_{n}^{(2)}=(-1)^{n+1} g\left(n+\frac{1}{2}\right) .
$$

The case $\mu=1$ of (14) yields $u_{n}=w_{n-1}-w_{n}$, therefore we get the following quasi-continuum approximations of the antisymmetric and symmetric breather profiles

$$
\begin{gathered}
u_{n}^{(1)}=(-1)^{n}[g(n)+g(n-1)], \\
u_{n}^{(2)}=(-1)^{n}\left[g\left(n+\frac{1}{2}\right)+g\left(n-\frac{1}{2}\right)\right] .
\end{gathered}
$$

The first graphs of figures 2 and 3 show the excellent agreement of these approximations with the numerical solutions of the stationary DpS equation. Returning to the ansatz (13) and the time-dependent (non-renormalized) DpS equation (12), we obtain approximate breather solutions of (9) taking the form

$$
y_{n}^{(s)}(t)=2 \epsilon u_{n}^{(s)} \cos \left(\omega_{\mathrm{b}} t\right), \quad \omega_{\mathrm{b}}=1+\frac{\epsilon^{1 / 2}}{2 \tau_{0}}, \quad s=1,2 .
$$

It is interesting to observe that approximation (20) is unaffected by smooth on-site nonlinear terms for $\epsilon \approx 0$, since we have noticed that the DpS equation remains unchanged.

In what follows we compare the above approximations with breather solutions of (9) computed numerically for free end boundary conditions. Let us note $Y_{n}=\left(y_{n}, \dot{y}_{n}\right)$. We use a method described in [10] to compute zeros $Y_{n}(0)=\left(y_{n}(0), 0\right)$ of the period map of the flow of $(9)$ (these initial conditions correspond to breathers even in time). The method of [10] is based on an adapted Gauss-Newton scheme and path-following.

An example of computation of a breather with frequency $\omega_{b}=1.1$ is shown in figure 4 . The initial guess used for the Newton method is the site-centered approximate breather solution derived from the DpS equation. After 5 iterations, the relative residual error

$$
E=\frac{\left\|\left\{Y_{n}\left(T_{b}\right)-Y_{n}(0)\right\}_{n}\right\|_{\infty}}{\left\|\left\{Y_{n}(0)\right\}_{n}\right\|_{\infty}}, \quad T_{b}=\frac{2 \pi}{\omega_{b}},
$$

reaches $1.5 \cdot 10^{-9}$ and the relative variation (measured using sup norms) of particle positions between successive iterates drops to $1.7 .10^{-11}$. Figure 4 compares the initial breather positions computed by the Newton method and their evolution at $t=100 T_{b}$, which shows that the breather oscillations are 
extremely stable. The super-exponential spatial decay of the breather is shown in figure 5.

Using the above numerical scheme, we obtain two branches of breather solutions of (9) with different symmetries, parametrized by their frequency $\omega_{b}>1$. They consist of bond-centered breathers, i.e. spatially antisymmetric solutions satisfying $y_{-n+1}=-y_{n}$ (figure 2) and site-centered breathers (figure 3 ). The latter possess subtle symmetry properties. Since the Hertz potential is noneven, equation (9) is not invariant by the symmetry $S y_{n}:=y_{-n}$. However, the set of $T_{b}$-periodic solutions of (9) is invariant under the transformation $S^{\prime} y_{n}(t)=-y_{-n}\left(t+T_{b} / 2\right)$. The site-centered breathers of (9) are left invariant by $S^{\prime}$ and not by $S$ (their asymmetry under $S$ increases with $\omega_{b}$ and becomes visible in the bottom panel of figure 3). On the contrary, the DpS equation admits both symmetries $S$ and $S^{\prime}$, which both leave the site-centered DpS breathers invariant.

These different types of symmetries are illustrated by figures 2 and 3, which compare the approximations (20) with breather solutions of (9) computed by the Newton method (middle and bottom plots). While approximation (20) is excellent at small amplitude (case $\omega_{b}=1.01$ ), its accuracy deteriorates in a more strongly nonlinear regime (case $\left.\omega_{b}=1.1\right)$.

More details on the continuation of discrete breathers in $\omega_{b}$ are shown in figure 6 , which compares the maximal amplitude and the energy of the bond-centered and site-centered breather solutions of (9) when $\omega_{b}$ is varied (the continuation is performed for $\left.\omega_{b} \in(1,2]\right)$. Both solutions bifurcate from $y_{n}=0$ when $\omega_{b} \rightarrow 1^{+}$, and their amplitude and energy increases with $\omega_{b}$. While minor differences between the breather amplitudes are visible, one observes that the respective energy curves are indistinguishable.

More generally, considering system (6) with the local anharmonic potential (10) and choosing $s \in[-1,1]$, we obtain branches of site-centered and bondcentered breathers bifurcating from the origin when $\omega_{b} \rightarrow 1^{+}$(results not shown). The persistence of both types of symmetries is due to the evenness of $W$.

In what follows we study in more detail the energy barrier separating sitecentered and bond-centered breathers. As illustrated below in section 3.3, this allows us to approximate the so-called Peierls-Nabarro energy barrier, which corresponds to the amount of energy required to put a stable static breather into motion under a momentum perturbation.

A notion of energy barrier separating discrete breathers is usually defined as follows (cf. also [52]). From (18)-(20), one can deduce a family of approximate 

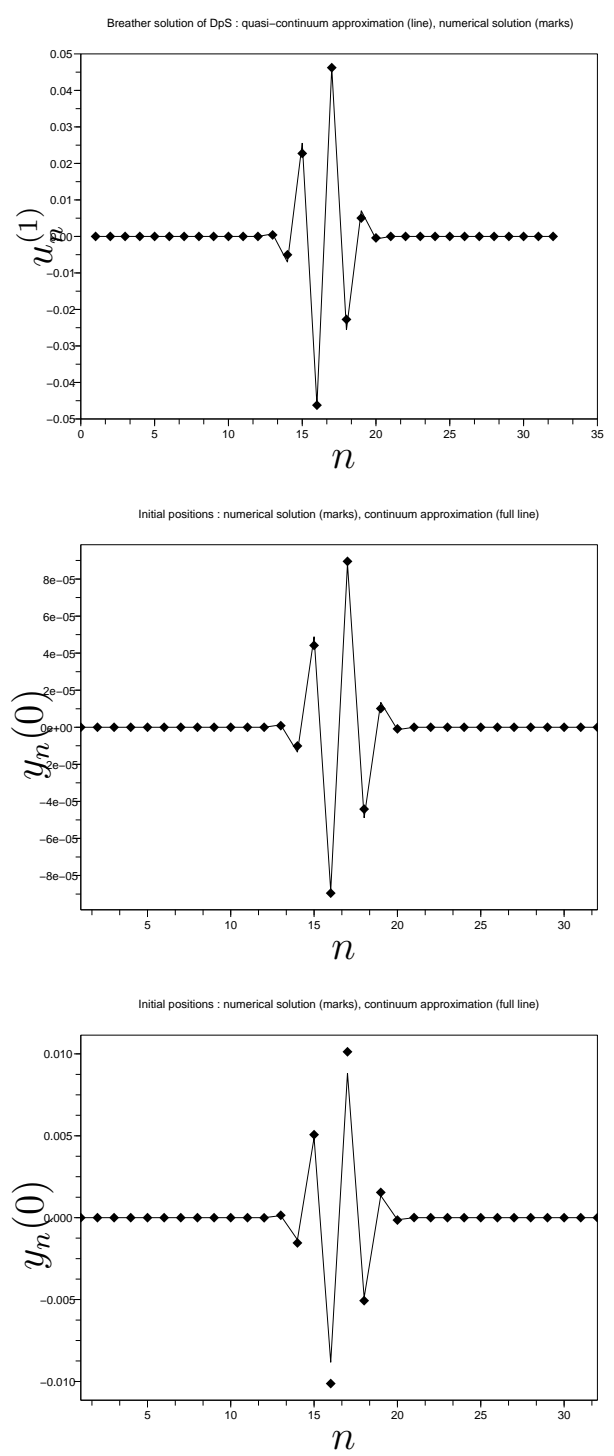

Fig. 2. Top panel: spatially antisymmetric solution of the stationary DpS equation (14), computed numerically for $\mu=1$ (marks). This solution is compared to the quasi-continuum approximation $u_{n}^{(1)}$ defined by equation (18) (continuous line). The other graphs compare a bond-centered breather solution of (9) computed numerically (marks) and its quasi-continuum approximation $y_{n}^{(1)}$ (continuous line). The middle plot corresponds to a small amplitude breather $\left(\omega_{b}=1.01\right)$, and the bottom plot to a more strongly nonlinear regime $\left(\omega_{b}=1.1\right)$.

static breather solutions of (6)-(10)

$$
y_{n}(t)=2 \epsilon\left[g\left(n+\frac{1}{2}-Q\right)+g\left(n-\frac{1}{2}-Q\right)\right](-1)^{n} \cos \left(\omega_{\mathrm{b}} t\right)
$$

where $\omega_{\mathrm{b}}=1+\frac{\epsilon^{1 / 2}}{2 \tau_{0}}$ and $Q \in \mathbb{R}$ (the cases $Q=0$ and $Q=1 / 2$ corresponding respectively to site-centered and bond-centered breathers). According to the works of $[52,44,65]$, approximate traveling breather solutions of (6)-(10) can be 

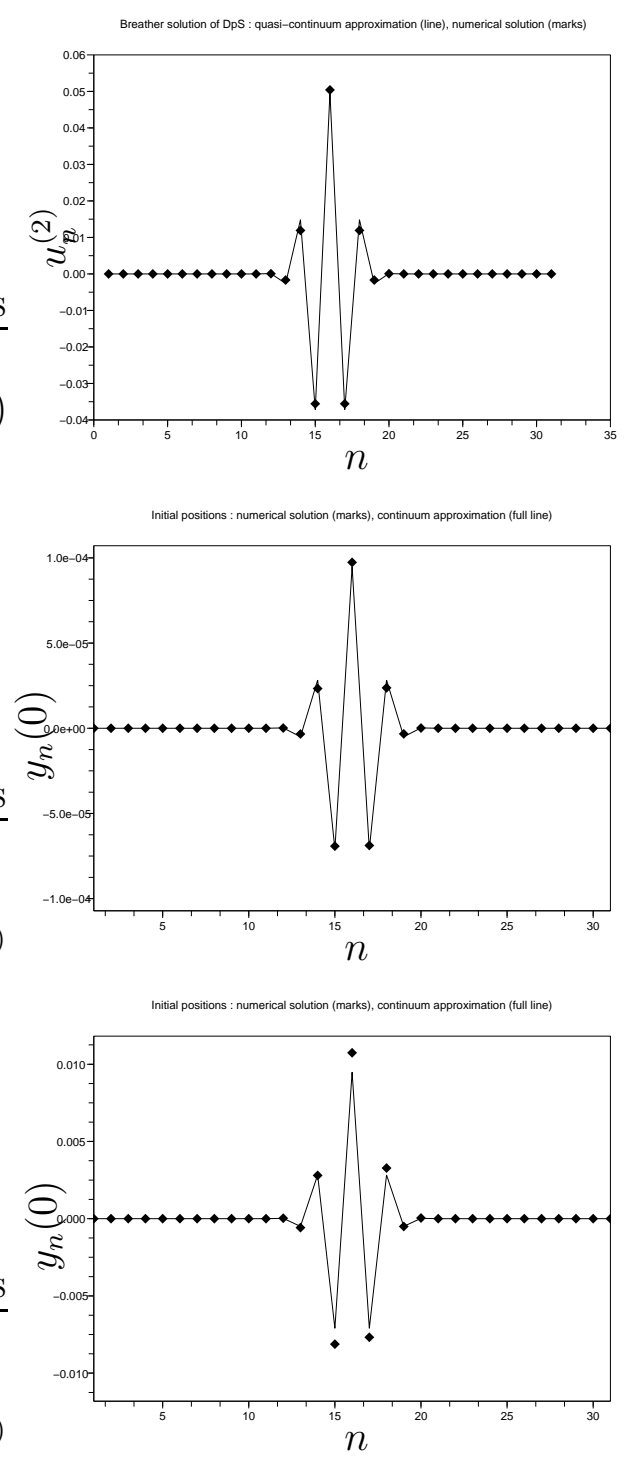

Fig. 3. Top panel: spatially symmetric solution of the stationary DpS equation (14), computed numerically for $\mu=1$ (marks). This solution is compared to the quasi-continuum approximation $u_{n}^{(2)}$ defined by equation (19) (continuous line). The other graphs compare a site-centered breather solution of (9) computed numerically (marks) and its quasi-continuum approximation $y_{n}^{(2)}$ (continuous line). The middle plot corresponds to a small amplitude breather $\left(\omega_{b}=1.01\right)$, and the bottom plot to a more strongly nonlinear regime $\left(\omega_{b}=1.1\right)$.

obtained from (21). Their dynamics is described by an effective Hamiltonian, whose critical points correspond to site-centered and bond-centered breathers having the same area

$$
\mathcal{A}=\int_{0}^{2 \pi / \omega_{b}} \sum_{n} \dot{y}_{n}^{2} d t
$$

The absolute energy difference $\tilde{E}_{P N}$ between the two breather solutions provides an approximation of the Peierls-Nabarro barrier. However, because the 


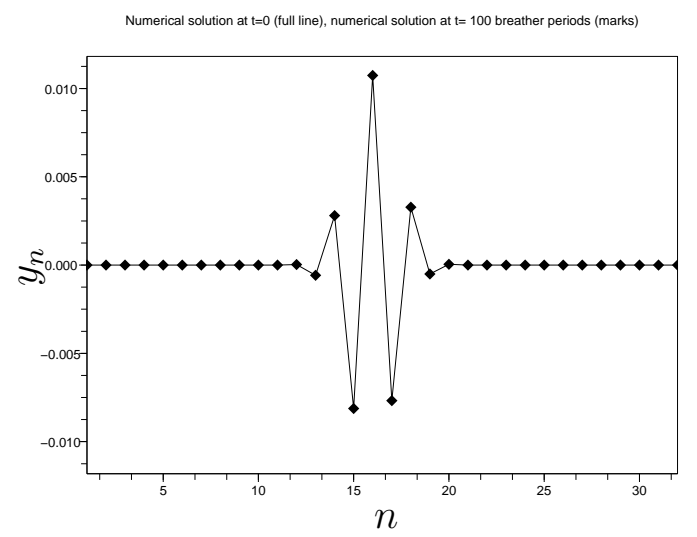

Fig. 4. Initial breather positions computed by the Newton method for a chain of 31 particles (full line), compared to their evolution at $t=100 T_{b}$ (marks). Computations are performed for a site-centered breather with frequency $\omega_{b}=1.1$.

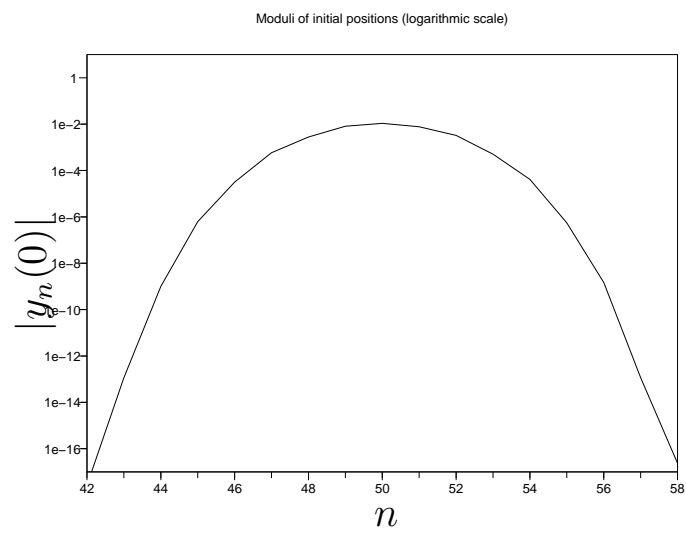

Fig. 5. Moduli of the initial breather positions computed by the Newton method, plotted in semi-logarithmic scale. Computations are performed for a chain of 99 particles and a site-centered breather with frequency $\omega_{b}=1.1$.

latter appears to be very small in system (6)-(10) (a phenomenon that will be illustrated in section 3.3 ), a very precise computation of $\tilde{E}_{P N}$ would be necessary. This yields additional numerical difficulties, due to the fact that the two breather frequencies have to be retrieved from a given area $\mathcal{A}$. Due to these difficulties, we shall use a more straightforward approach and define (following ref. [14]) the approximate Peierls-Nabarro barrier $E_{P N}$ as the absolute energy difference between site-centered and bond-centered breathers having the same frequency $\omega_{b}$.

We obtain extremely small values of $E_{P N}$ both for harmonic and anharmonic on-site potentials, even quite far from the small amplitude regime. This result is illustrated by figure 7 for $s=-1 / 6, s=0$ and $s=1$. For small amplitude breathers $\left(\omega_{b} \approx 1.01\right.$ in our computations), the different values of $s$ yield comparable values of $E_{P N}$, of the order of $10^{-14}-10^{-15}$. We find that $E_{P N}$ 

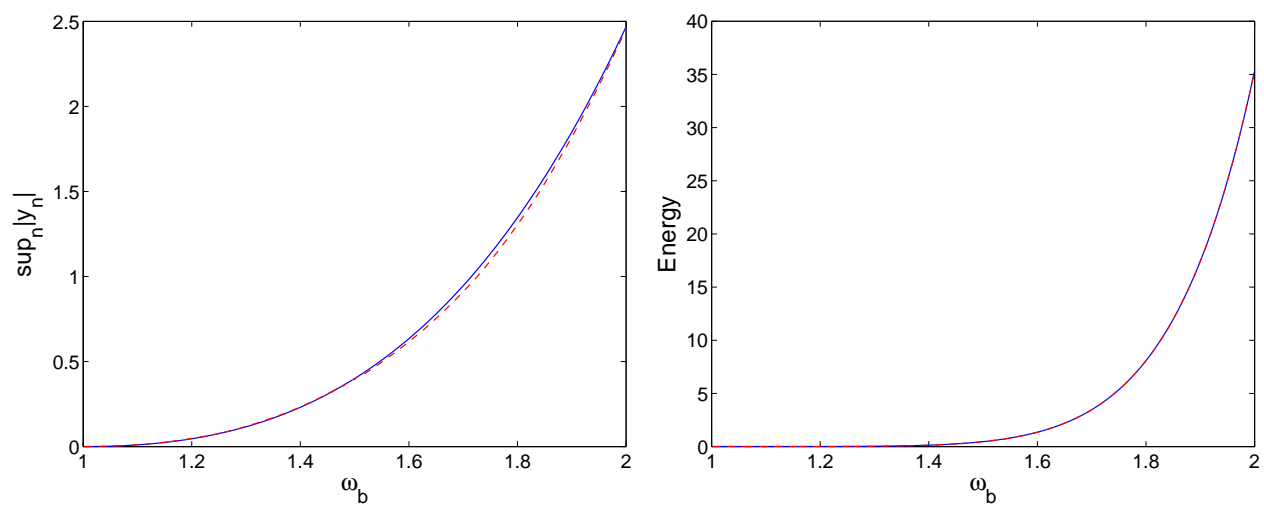

Fig. 6. Maximal amplitude at $t=0$ (left plot) and energy (6) (right plot) of breather solutions of (9) as a function of their frequency $\omega_{b}$. The continuous line corresponds to bond-centered breathers, and the dashed line to site-centered breathers. Notice that the energy curves are indistinguishable.

increases with the breather amplitude but remains very small in our parameter range (e.g. $E_{P N}$ is close to $10^{-4}$ for $\omega_{b}=1.5$ and $s=-1 / 6$ ). The harmonic case yields even much smaller barriers (by $3-4$ orders of magnitude for $\omega_{b}=1.3$ ). As shown by figure 7 , the smaller relative energy difference between sitecentered and bond-centered breathers is also achieved in the harmonic case. These results indicate that extremely small perturbations of the breathers are capable of putting them into motion (even more critically for harmonic on-site potentials), a phenomenon that will be illustrated in the next section.

\subsection{Breather stability and mobility}

In this section we examine the stability properties of spatially antisymmetric and symmetric breather solutions of (11) and (6), and link these properties with the existence of traveling breather solutions. The linear (spectral) stability of breather solutions of (11) is investigated by means of the perturbation [45]:

$$
v_{n}(t)=\exp (i \mu t)\left[u_{n}+\left(a_{n} \exp (\lambda t)+b_{n}^{\star} \exp \left(\lambda^{\star} t\right)\right)\right]
$$

where $u_{n}$ is a spatially symmetric or antisymmetric solution of (14) homoclinic to 0 . The resulting linear problem for the eigenvalue $\lambda$ and the eigenvector $\left(a_{n}, b_{n}\right)^{T}$ (where ${ }^{T}$ denotes transpose) is solved by standard numerical linear algebra solvers and the results are depicted by means of the spectral plane $\left(\lambda_{r}, \lambda_{i}\right)$ of the eigenvalues $\lambda=\lambda_{r}+i \lambda_{i}$.

From the bottom panels of Fig. 8, we can infer that spatially antisymmetric solutions are spectrally stable and therefore should be structurally robust (a result confirmed by our direct numerical simulations - data not shown here--). 

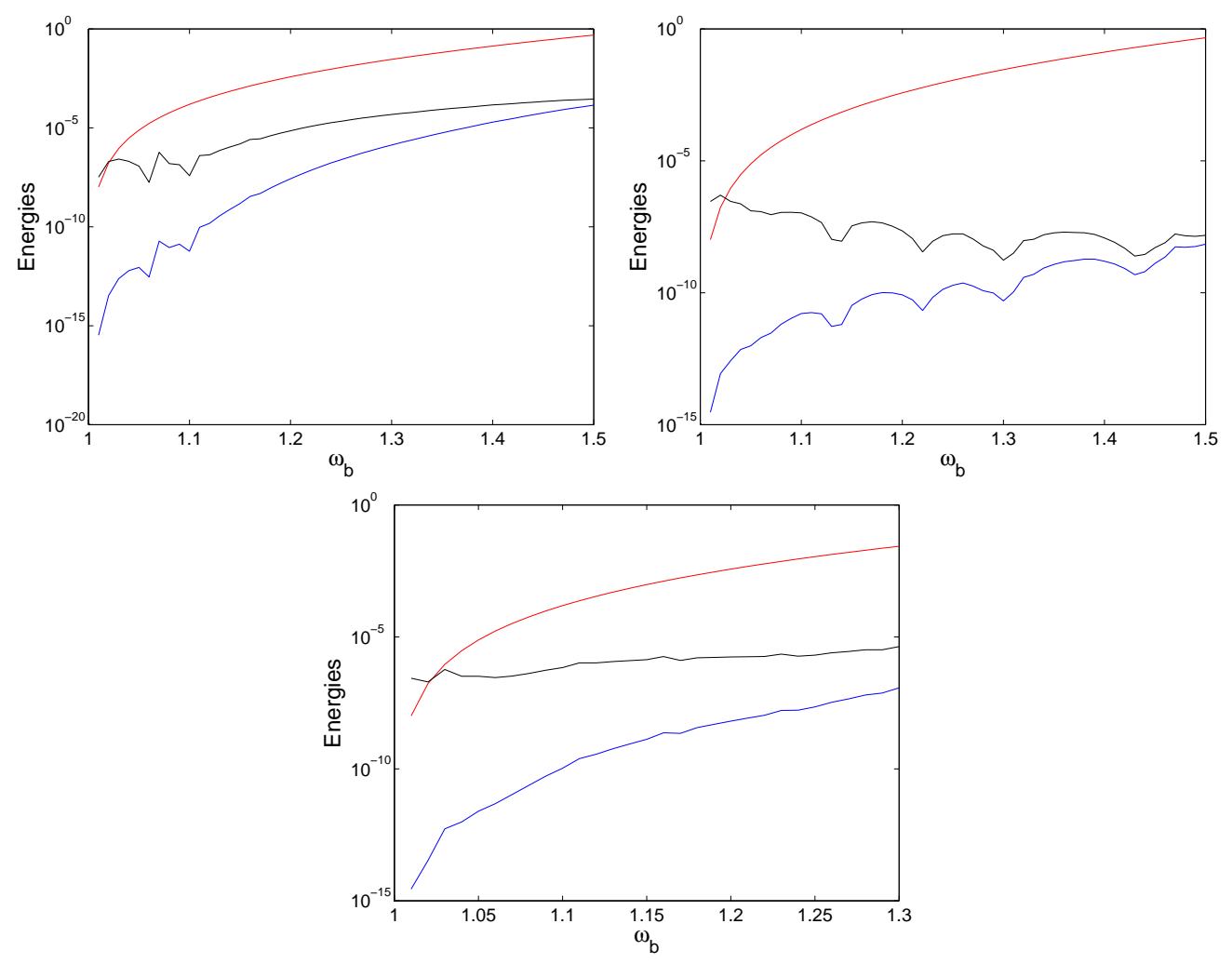

Fig. 7. Approximate Peierls-Nabarro barrier computed as a function of breather frequency, for different degrees of anharmonicity of the on-site potential (top left plot : $s=-1 / 6$, top right plot $: s=0$, bottom plot $: s=1$ ). The red curves give the energy $E_{b c}$ of bond-centered breathers defined by (6). The blue curves correspond to the approximate Peierls-Nabarro barriers $E_{P N}$ (see text), and the black curve to the relative energy ratio $E_{P N} / E_{b c}$ between the energy barrier and the bond-centered breather energy.

This is due to the absence of eigenvalues of non-vanishing real part in this Hamiltonian system (in which whenever $\lambda$ is an eigenvalue, so are $\lambda^{\star},-\lambda$ and $\left.-\lambda^{\star}\right)$.

On the other hand, the stability and associated dynamical properties are more interesting in the case of the site-centered solution of Fig. 9. In this case, we can observe the presence of a real eigenvalue pair. As can be seen in the bottom panel of Fig. 9, the real part of the relevant eigenvalue pair (which corresponds to the instability growth rate) grows linearly with the eigenvalue parameter $\mu$, inducing a progressively stronger instability for larger amplitude solutions. The dynamical manifestation of this instability is illustrated in Figure 10. Here we perturb the dynamically unstable solution of the right panel of Fig. 9 by a uniformly distributed random perturbation (of amplitude 0.01). The projection of this random field on the unstable eigenvector of the site centered mode excites the manifestation of the dynamical instability of this mode which is, in turn, illustrated in the space-time evolution (where the colorbar corresponds to the field $\left|v_{n}(t)\right|^{2}$ ) of Fig. 10. Clearly, the instability of 

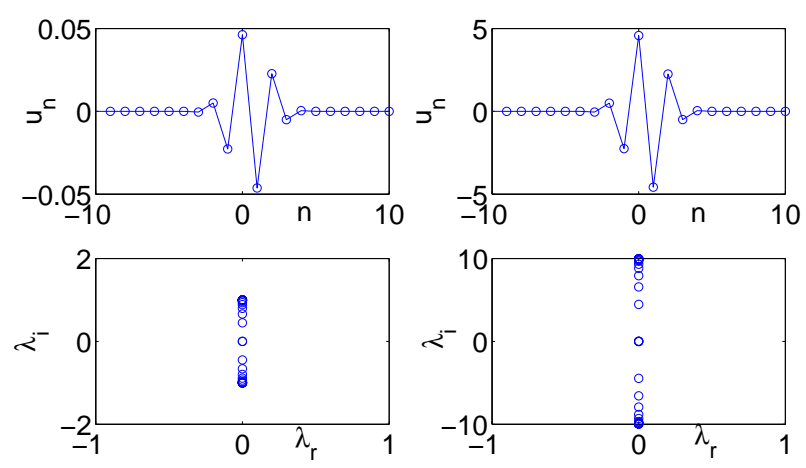

Fig. 8. The profiles (top panels) and the linear stability (bottom panels) of the spatially antisymmetric solution of the $\mathrm{DpS}$ equation are shown for the values of $\mu=1$ (left panels) and $\mu=10$ (right panels). This inter-site solution is linearly stable.

the site-centered mode is associated with a "translational" eigenmode of the linearization problem, whose excitation induces the motion of the localized mode.

In the above analysis, the breather stability properties remain qualitatively unchanged for all values of $\mu$. This follows from the scale invariance of (11) pointed out in section 3.2, which also implies the linear dependence of the eigenvalues $\lambda$ on $\mu$. However, we note in passing that this simplification is obviously not valid for the model (9).

Having determined the spectral stability of bond-centered and site-centered breather solutions in the DpS equation, we now consider the same problem for their analogues in the original lattice (6), including in our analysis the effect of a possible addition of a local anharmonic potential (10).

We have computed the Floquet spectrum of (6)-(10) linearized at the bondcentered breather and the site-centered breather, for different values of the breather frequency $\omega_{b} \in(1,2]$ and the anharmonicity parameter $s \in[-1,1]$. The Floquet spectrum includes a quadruplet of eigenvalues close to +1 and eigenvalues on the unit circle accumulating near $e^{ \pm i 2 \pi / \omega_{b}}$. The spectral properties of these discrete breathers differ from usual ones [54] for several reasons. Firstly, no bands of continuous spectrum are present on the unit circle for the infinite chain. This is due to the fact that system (9) linearized at $y_{n}=0$ (the limit of a breather solution at infinity) consists of an infinite chain of uncoupled identical linear oscillators, and thus the phonon band reduces to a single frequency, equal to unity in the present case. Secondly, another nonstandard property originates from the quadruplet of eigenvalues close to +1 . Due to the Hamiltonian character of $(9),+1$ is always at least a double eigenvalue of the Floquet matrix. In addition, we always find an extra pair of eigenvalues in the immediate vicinity of +1 corresponding to a pinning mode (see below). 

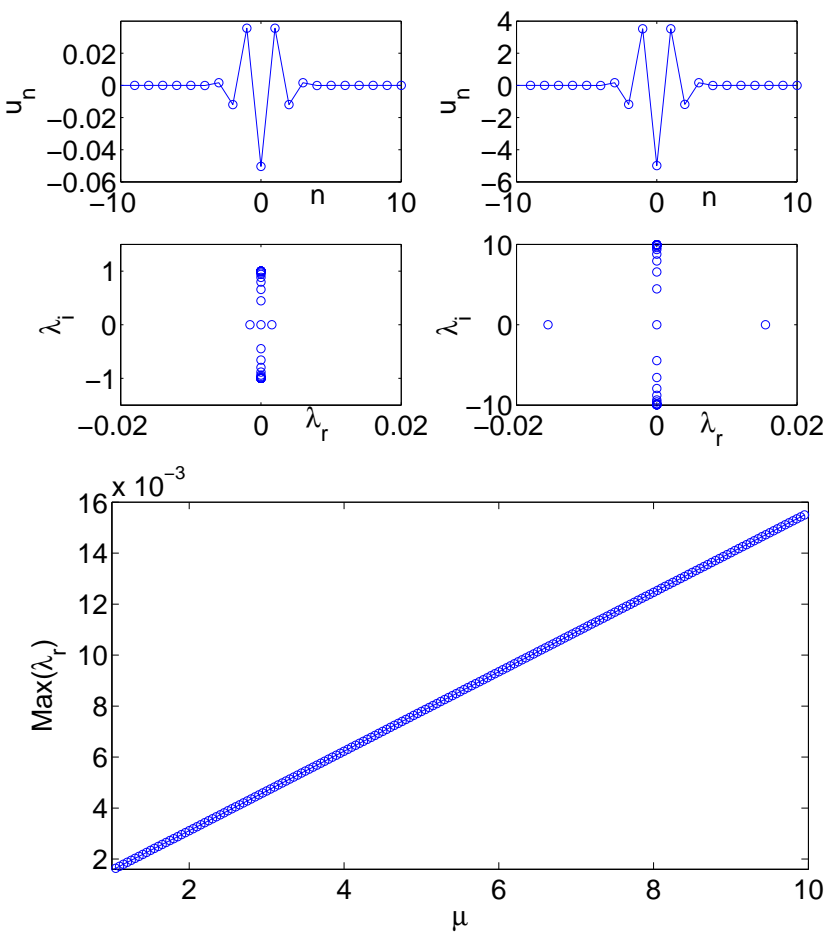

Fig. 9. The top panel is directly analogous to the results of Fig. 8, but for the case of the site-centered solution. The presence of a real eigenvalue pair of linearly growing magnitude as $\mu$ increases can be observed in the spectral plane and is more clearly highlighted in the figure of the bottom panel.

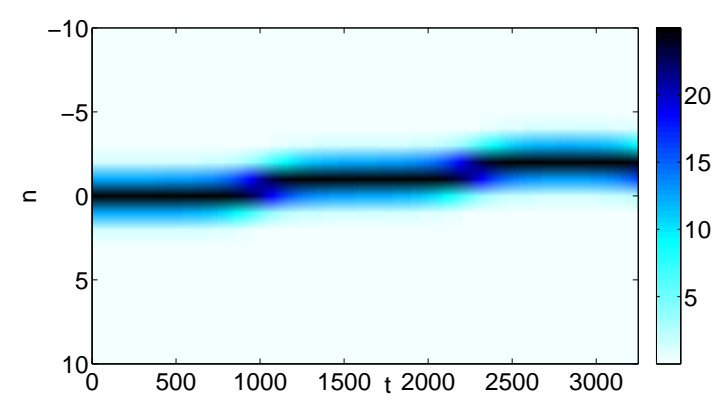

Fig. 10. The figure shows the space-time contour plot of the square modulus of the field $\left|v_{n}(t)\right|^{2}$ for equation (11). The initial condition is a site-centered localized mode (from the right panel of Fig. 9), perturbed by a uniformly distributed random perturbation of amplitude 0.01 . The perturbation leads to the manifestation of the instability of the site-centered mode which, in turn, leads to its mobility.

This contrasts with the case of Klein-Gordon lattices, where this situation is a codimension-one phenomenon, occuring near critical values of the coupling constant and for particular classes of on-site potentials $[2,9,4]$.

In what follows we describe the evolution of the quadruplet of eigenvalues close to +1 for $\omega_{b}=1.1$ and $s \in[-1,1]$. The following figures display the moduli and arguments of these eigenvalues for the bond-centered breather (figure 11) 
and the site-centered breather (figure 12). For the bond-centered breather, a pair of Floquet multipliers $\lambda, \lambda^{-1}$ emerges from the unit circle after a collision at +1 , for $s>s_{0}^{b} \approx 0.26$. For the site-centered breather, a pair of multipliers $\lambda, \lambda^{-1}$ (with $\lambda>1$ ) exists for $s<s_{0}^{s} \approx 0.05$, and enters the unit circle for $s>s_{0}^{s}$ after a collision at +1 .
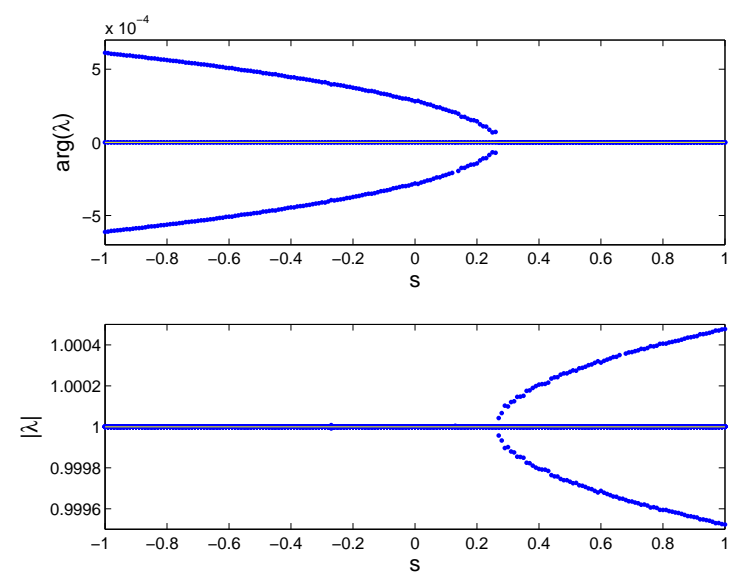

Fig. 11. Arguments (upper plot) and moduli (lower plot) of the quadruplet of Floquet eigenvalues $\lambda$ close to +1 , corresponding to system (9)-(10) linearized at the bond-centered breather. Computations are performed for $\omega_{b}=1.1$, and eigenvalues are plotted as a function of the anharmonicity parameter $s \in[-1,1]$.
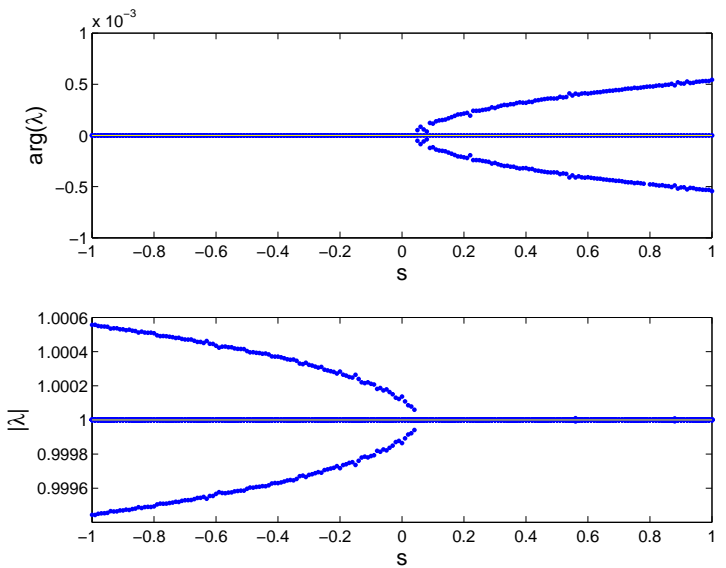

Fig. 12. Same plot as in figure 11, for the site-centered breather with $\omega_{b}=1.1$.

From the above spectral study, one can infer that for harmonic on-site potentials (i.e. $s=0$ ) and $\omega=1.1$, the site-centered breather is weakly unstable and the bond-centered breather is spectrally stable. These results agree with the above results obtained for the DpS equation. This provides a consistent picture, given that the $\mathrm{DpS}$ equation correctly approximates breather profiles of amplitudes $\epsilon=O\left(\left(\omega_{b}-1\right)^{2}\right)$ for $\omega_{b} \approx 1$ (section 3.2). The DpS admits weakly unstable site-centered and stable bond-centered breather solutions, and approximates the dynamics of $(9)$ for $O(\epsilon)$ initial data on times of order $O\left(\epsilon^{-1 / 2}\right)$ [18]. Hence, we expect a parallel to the instability of site-centered 
modes of the DpS dynamics in Eq. (9). Note that these instabilities are extremely small for $\omega_{b}$ close to 1 , because the instability of the site-centered breather is already very weak at the renormalized (slow) time-scale of the DpS equation (see figure 9 ), and becomes $O\left(\epsilon^{1 / 2}\right)$ times weaker at the level of (9) for a breather with amplitude $\epsilon$.

The above picture persists for $s \approx 0$, but the site-centered and bond-centered breathers display a change of stability at the two different critical values $s=$ $s_{0}^{b, s}>0\left(s_{0}^{s}\right.$ being quite small), after which their dynamical stability differs from the stability of the DpS breathers. It would be interesting to analyze the bifurcations of new types of discrete breathers near these critical values of $s$, and this problem will be considered in a future work.

In what follows we illustrate the effect of the additional Floquet eigenvalues close to +1 on the breather dynamics, again considering the case $\omega_{b}=1.1$. Figure 13 compares an eigenvector associated with one of these eigenvalues and the renormalized discrete gradient

$$
g_{n}=\frac{y_{n+1}(0)-y_{n-1}(0)}{\sum_{n}\left|y_{n+1}(0)-y_{n-1}(0)\right|^{2}}
$$

which reveals that the two profiles are very close. The associated mode will thus be referred to as a translation mode or pinning mode, and the effect of a perturbation along its direction is to shift the breather center [9]. The existence of this mode has the effect of enhancing the breather mobility. To illustrate this, we perturb at $t=0$ the velocity components of a stationary breather, adding the discrete gradient $g_{n}$ multiplied by a velocity factor $c$. The kinetic energy imprinted to the lattice is then $c^{2} / 2$. We consider below the energy density at the $n$-th site, which is defined from (6):

$$
e_{n}=\frac{1}{2} \dot{y}_{n}^{2}+W\left(y_{n}\right)+\frac{2}{5} \gamma\left(y_{n}-y_{n+1}\right)_{+}^{5 / 2}
$$

Fig. 14 shows the energy density plot in the system of Eqs. (6)-(8), for a sitecentered and a bond-centered breather perturbed with $c=2 \times 10^{-4}$. This perturbation results in a translational motion of the breather at an almost constant velocity with negligible dispersion, which illustrates the strong mobility of discrete breathers in the present model. These results are consistent with the approximation $E_{P N}$ of the Peierls-Nabarro barrier computed previously, since we found $E_{P N} \approx 1.77 .10^{-11}$ for $s=0$ and $\omega_{b}=1.1$ (see figure 7). The above momentum perturbation increases the kinetic energy of the bond-centered breather by $c^{2} / 2=2 \times 10^{-8}$, which is well-above $E_{P N}$.

To describe the effect of breather perturbations below the Peierls-Nabarro 
barrier, it is convenient to consider the breather energy center

$$
X=\frac{\sum_{n=n^{\prime}-m}^{n^{\prime}+m} n e_{n}}{\sum_{n=n^{\prime}-m}^{n^{\prime}+m} e_{n}}
$$

with $n^{\prime}$ being the location of the maximum energy density of the breather and $m>0$ an integer which accounts for the width of the breather (we have fixed $m=5)$. Figure 15 displays $X(t)$ for $c=3.10^{-6}$, i.e. $c^{2} / 2=4.5 \times 10^{-12}$ lying below $E_{P N}$. In that case, only the unstable site-centered breather is able to move along the lattice (it is able to jump 2 sites but gets pinned subsequently). For the stable bond-centered breather, a transition from pinning to mobility is obtained for $c>c_{c} \approx 6.19 \cdot 10^{-6}$. The value of the Peierls-Nabarro barrier resulting from dynamical simulations is thus $c_{c}^{2} / 2 \approx 1.92 .10^{-11}$, which is quite close to the approximation $E_{P N}$ computed previously.
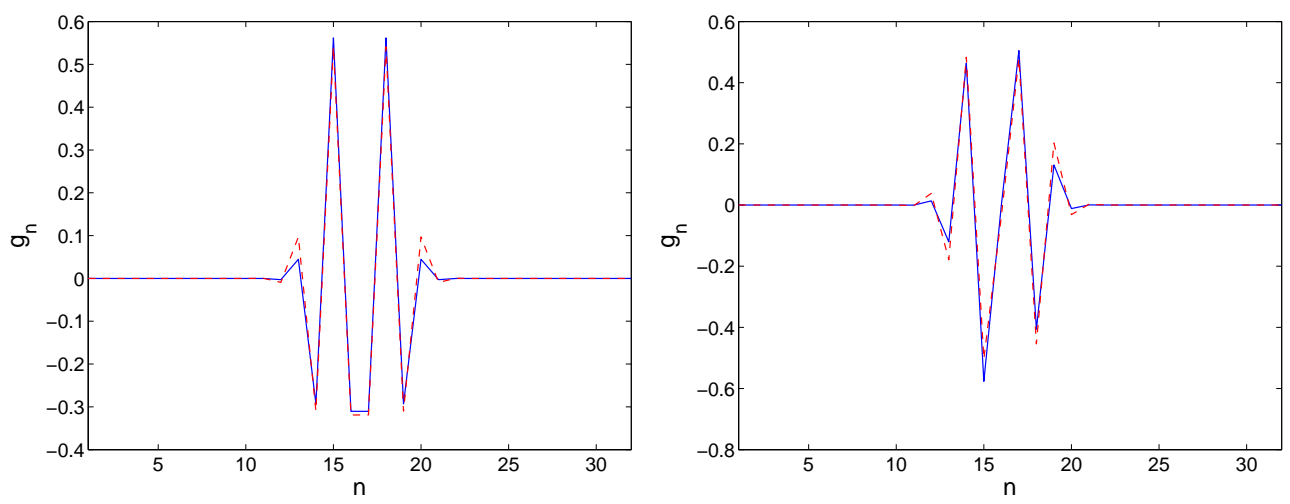

Fig. 13. Pinning mode (full line) and discrete gradient (dashed line) corresponding to a bond-centered (left plot) and site-centered (right plot) stationary breather in system (9), for the breather frequency $\omega_{b}=1.1$. The components of the pinning mode correspond to particle positions at $t=0$ (initial particle velocities vanish).

\subsection{Study of an impact problem}

Having demonstrated the mobility of breather modes in the DpS equation, in direct analogy with the dynamics of the full oscillator model, we attempt the excitation of the first site of a Newton's cradle and the associated DpS chain, and observe the ensuing space-time evolution.

Consider the equation (12) on a semi-infinite lattice with $n \geq 1$ and a free end boundary condition at $n=1$. We numerically compute the solution of (12) with the initial condition

$$
A_{1}(0)=-i, A_{n}(0)=0 \text { for } n \geq 2 \text {. }
$$

It can be clearly seen in Fig. 16 that the result is the formation of a localized 

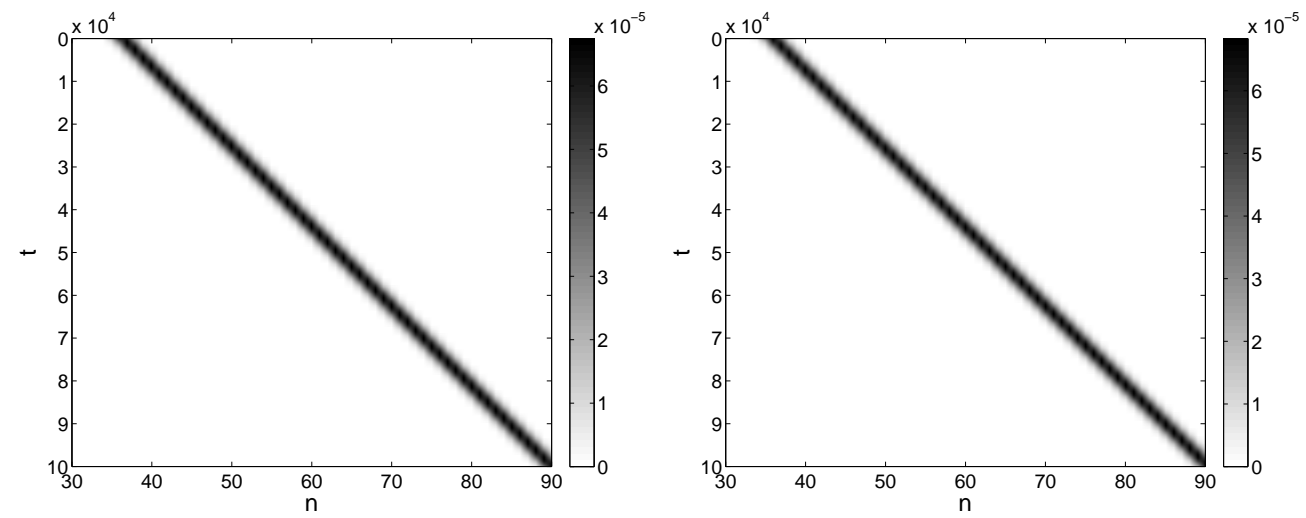

Fig. 14. Energy density plot of a moving breather in system (6)-(8), obtained by perturbing along the pinning mode a bond-centered (left plot) and site-centered (right plot) stationary breather with frequency $\omega_{b}=1.1$. The initial velocity perturbation has a magnitude $c=2 \times 10^{-4}$. The velocities $v$ of resulting traveling breathers are very close, i.e. $v \approx 5.446 .10^{-4}$ for the site-centered case and $v \approx 5.364 .10^{-4}$ for the bond-centered case, resulting in nearly identical figures.

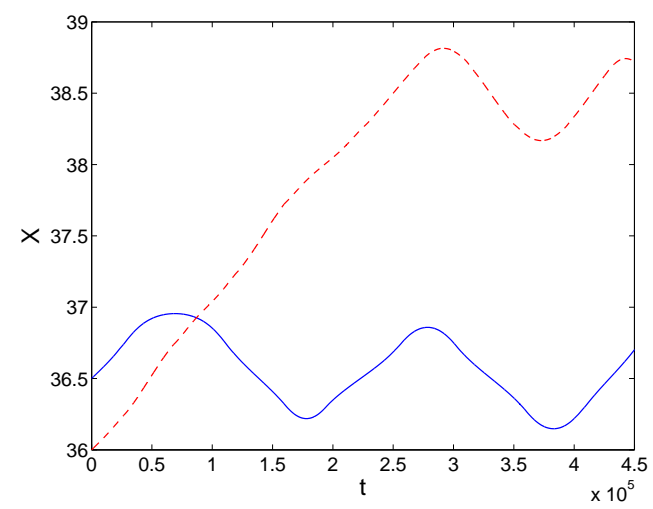

Fig. 15. Time-evolution of the breather energy center after a momentum perturbation of a bond-centered (full line) and site-centered (dashed line) breather. All parameters are the same as in figure 14, except the initial velocity perturbation $c$. We fix $c=3.10^{-6}$, which corresponds to an increase of kinetic energy below the Peierls-Nabarro barrier.

excitation which is traveling robustly through the chain. This is the traveling breather resulting from the mobility of the discrete breathers that we considered before. In addition to this strongly localized nonlinear excitation, we can observe a weak residual excitation at the end of the chain (this is somewhat reminiscent of the phenomenology described in [32]). Strictly speaking, we cannot consider this mode to be a surface mode of the chain [57], as our observations indicate that its profile is fairly extended and non-stationary (or periodic).

For all $\epsilon>0$ small enough, the above solution of DpS corresponds to an approximate solution of (9) given by (13), satisfying $y_{n}^{\text {app }}(0)=0, \dot{y}_{1}^{\text {app }}(0)=$ 


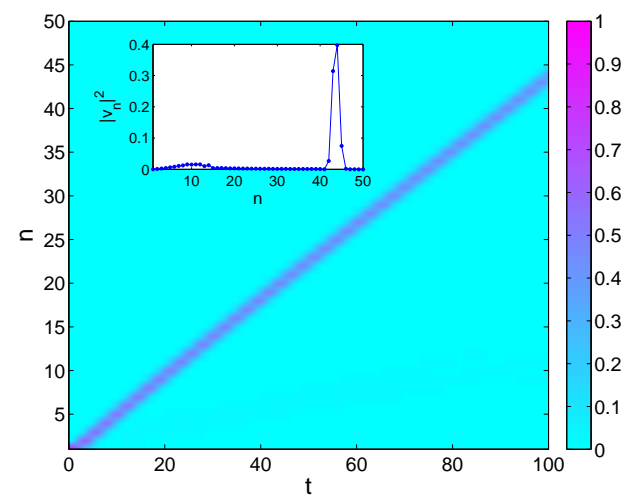

Fig. 16. The space-time evolution of the square modulus of the field, similarly to Fig. 10, under the DpS equation is shown for an initial excitation of the domain boundary site with $A_{n}(0)=-i \delta_{n, 1}\left(\delta_{i, j}\right.$ denotes the usual Kronecker symbol). The corresponding spatial profile is depicted in the inset for the final shown simulation time. Notice the robust propagating localized mode (traveling breather), as well as the presence of a weak residual (fairly extended) excitation near the boundary.

$2 \epsilon+O\left(\epsilon^{3 / 2}\right), \dot{y}_{2}^{\text {app }}(0)=O\left(\epsilon^{3 / 2}\right)$ and $\dot{y}_{n}^{\text {app }}(0)=0$ for $n \geq 3$. Figures 17 and 18 compare the corresponding approximate solution (13) and the solution of (9) with initial condition

$$
y_{n}(0)=0, \quad \dot{y}_{1}(0)=2 \epsilon, \quad \dot{y}_{n}(0)=0 \text { for } n \geq 2
$$

for a small value of $\epsilon$. One can see that the DpS equation and the full oscillator model give rise to similar dynamics, i.e. the initial impulse splits into a traveling breather and an extended wavetrain emitted from the boundary. The amplitude of the latter is reasonably well reproduced by the DpS approximation over a long transient, while the traveling breather amplitude and velocity are overestimated (e.g., the breather amplitude is approximately $13 \times 10^{-4}$ with the DpS approximation and $9 \times 10^{-4}$ for the full lattice). In addition, the traveling breather velocity resulting from the DpS approximation is slightly larger than in the full oscillator model. The same kind of waves are visible up to moderate initial velocities. The traveling breather remains highly localized (mainly supported by 7 lattice sites), and is followed by a small oscillatory tail reminiscent of the periodic traveling waves analyzed in [41].

In what follows we analyze the effect of considering the local anharmonic potential (10). Due to the smoothness of $W$, the DpS equation remains unchanged with respect to the harmonic case, as observed in section 3.1. Consequently, the dynamics of (9) after the impact is expected to remain unchanged for small excitations, on the time scales given in section 3.1. However, it is interesting to examine possible additional effects of anharmonicity occuring on longer time scales or for large amplitude excitations. For example, a trapping of large amplitude traveling breathers can occur in Klein-Gordon lattices $[14,52]$, due to the Peierls-Nabarro energy barrier separating site-centered and 

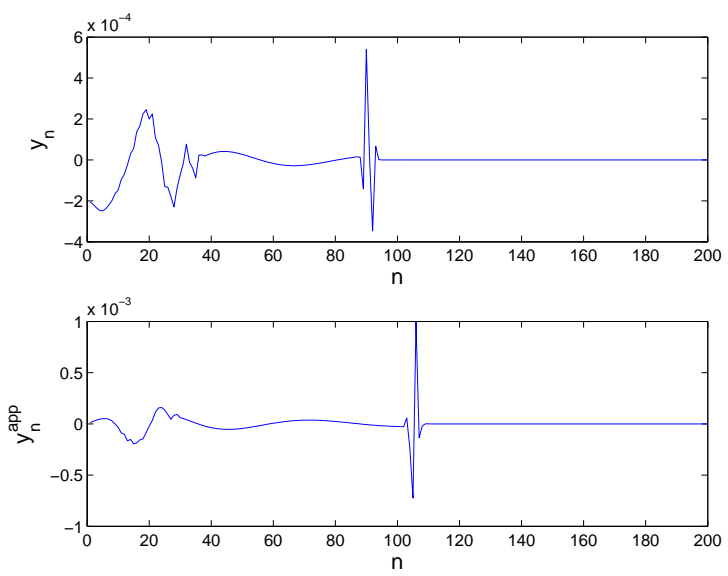

Fig. 17. Comparison between the solution of (9)-(26) (upper plot) and its approximation given by (12)-(13)-(25) (lower plot), at a fixed time $t \approx 8090$ corresponding to $\tau=250$. Computations are performed for $\epsilon=0.9548 .10^{-3}$. For this value of $\epsilon$, the static breathers given by (20) have frequency 1.01, and the DpS equation yields very good approximations of the static breathers.
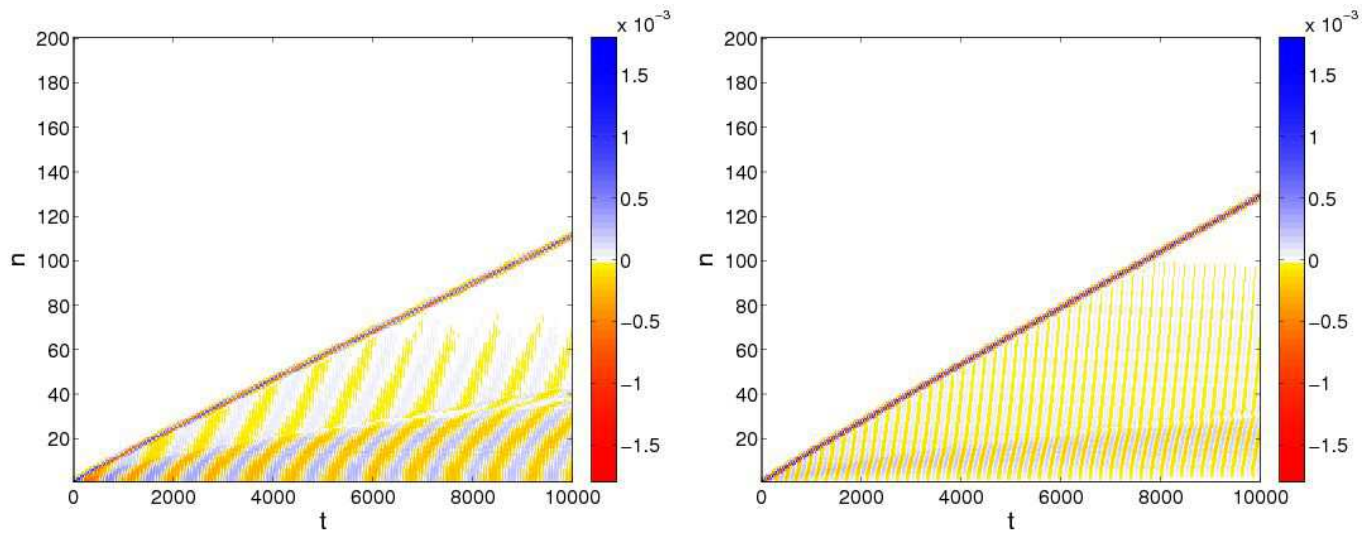

Fig. 18. Energy density plot showing the comparison between the solution of (9)-(26) (left plot) and its approximation given by (12)-(13)-(25) (right plot), for $\epsilon=0.9548 \times 10^{-3}$. Time $t$ in the bottom plot is related to the slow time $\tau$ through $t=\epsilon^{-1 / 2} \tau$.

bond-centered breathers.

In order to characterize the breather motion we consider the traveling breather energy center $X(t)$ defined by (24). The average velocity of the traveling breather is computed as the slope of the linear least squares approximation of the function $X(t)$, taking only into account the points for which the traveling breather is sufficiently far from the boundary in order to eliminate boundary effects. Figure 19 displays the traveling breather velocity and maximum energy density (computed from $(23)$ ) as a function of the initial velocity $\dot{y}_{1}(0)$, for different values of the parameter $s \leq 0$. As expected, the different graphs are very close at small initial velocity where the DpS equation drives the dynamics, but discrepancies appear at larger velocities. The graphs of figure 19 corresponding 
to $s<0$ are interrupted above some critical velocities, because the solution blows-up in finite time when the initial velocity exceeds some threshold. Below this value, the anharmonicity of the on-site potential with $s<0$ decreases the breather velocity. The energy of the traveling breather (including its kinetic energy) becomes much smaller because a part of the initial energy remains trapped in the form of a surface mode located near $n=1$. This phenomenon is illustrated in figure 20, which compares the traveling breather propagation in two chains with $s=0$ (left plot) and $s=-0.7$ (right plot), for $\dot{y}_{1}(0)=0.94$. In the left panel, a highly-localized traveling breather is clearly visible, while the right panel shows a surface mode and a lower-energy traveling breather with smaller velocity. Typical profiles of the surface mode and the traveling breather are displayed in figure 21 . The possibility of exciting a surface mode by an impact was already pointed out in reference [15], for a mixed KleinGordon - FPU chain with a sinusoidal local potential, and a Morse interaction potential instead of the fully-nonlinear Hertzian interactions.
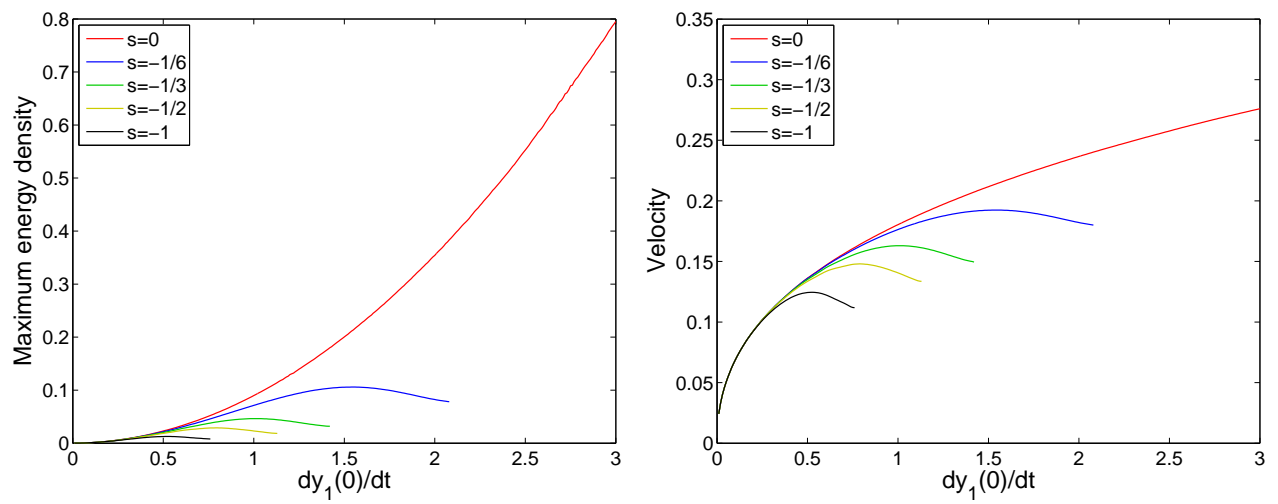

Fig. 19. Maximum energy density (left plot) and velocity (right plot) of the traveling breather generated in system (6)-(10) with initial condition (26), for several values of $\dot{y}_{1}(0)$ and anharmonicity parameter $s \leq 0$.

Note that the above-mentioned blow-up phenomenon is due to potential (10) with $s<0$ and does not occur for $W(y)=1-\cos y$, which corresponds e.g. to the gravitational potential acting on the usual Newton's cradle. In the latter case, the dynamics resulting from the impact becomes rather similar to the phenomena studied in [15]. For sufficiently large impact velocities the traveling breather is replaced by a kink reminiscent of Nesterenko's soliton [58], resulting in the ejection of a finite number of particles at the end of the chain (result not shown). It would be interesting to analyze how the transition between traveling breather and kink excitations occurs in this system, but this problem lies beyond the scope of the present paper.

In the case $s>0$ of (10) we observe a different scenario illustrated by figure 22. The traveling breather doesn't move at constant velocity, but instead behaves like a "bouncing ball" against the boundary at $n=1$, i.e. it experiences alternating phases of deceleration, direction-reversing, accelerated backward 

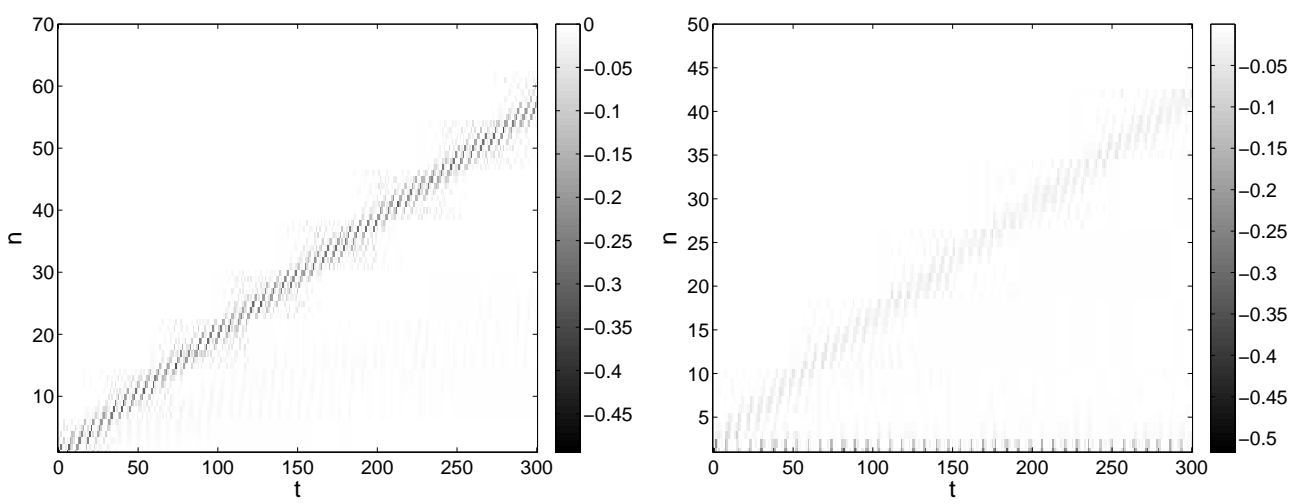

Fig. 20. Space-time diagrams showing the interaction forces $f_{n}=-\left(y_{n}-y_{n+1}\right)_{+}^{3 / 2}$ in system (6)-(10) for the initial condition $(26)$ with $\dot{y}_{1}(0)=0.94$. Forces are represented in grey levels, white corresponding to vanishing interactions (i.e. beads not in contact) and black to a minimal negative value of the contact force. Several values of the anharmonicity parameter are considered $: s=0$ (left plot), $s=-0.7$ (right plot).

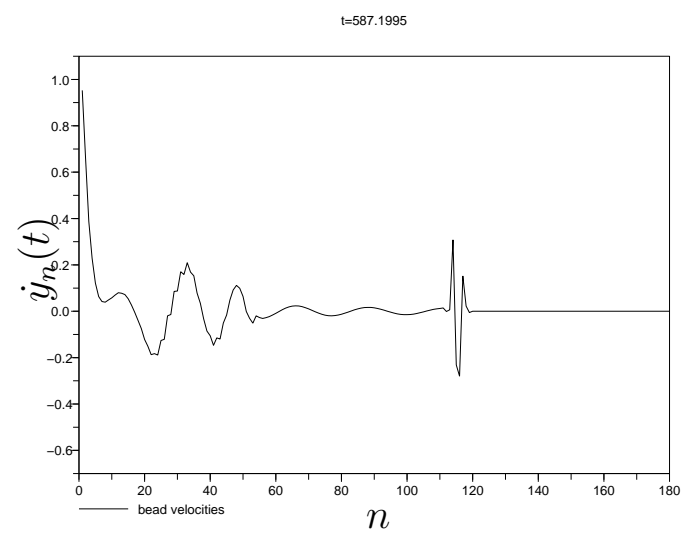

Fig. 21. Snapshot of particle velocities in system (6)-(10) with anharmonicity parameter $s=-1 / 6$. The profile is plotted at $t \approx 587$, for the initial condition (26) with $\dot{y}_{1}(0) \approx 1.87$. The excitations of a surface mode and a traveling breather are clearly visible.

motion towards the boundary, and rebound at the boundary (bottom panel of figure 22). During a few rebounds, the breather center behaves like a Newtonian particle in an almost constant effective force field. This phenomenon seems to take place as soon as $s>0$ (we have checked it for $s \geq 0.005$ ), but not for $s=0$. The effective force field both increases with $s$ and with the imprinted initial velocity. For moderate initial velocities the traveling breather deceleration is quite slow, as shown by the top panel of figure 22. Figure 23 displays a traveling breather profile at the onset of direction-reversing. These traveling breathers with direction-reversing motion are reminiscent of excitations known as "boomerons", consisting of direction-reversing solitons discovered in different kinds of integrable models (see [16] and references therein), but the link between both phenomena remains quite speculative at this stage. Although we 
have no clear explanation of the origin of direction-reversing for the traveling breather, one possibility might be its interaction with other nonlinear waves visible in figure 23, which are confined between the traveling breather and the boundary. In addition, the rebound dynamics can be followed by phases of intermittent trapping or erratic motion of the breather (figure 22, middle panel).

\subsection{Dimensional analysis of the $D p S$ limit}

The DpS limit has been previously described for equation (9) written in a normalized form. In this section, we consider a general class of granular systems with on-site potentials and use suitable scalings to rewrite the system in the form (9). Returning to the above impact problem, this allows us to analyze in which parameter regime the DpS equation drives the dynamics. As we shall see, this case occurs when local oscillations are faster than binary collisions.

We consider a chain of identical beads of mass $m$ sitting in local harmonic potentials, described by the Hamiltonian

$$
\mathcal{H}=\sum_{n} \frac{m}{2} \dot{x}_{n}^{2}+\frac{k}{2} x_{n}^{2}+\frac{2}{5} \gamma\left(x_{n}-x_{n+1}\right)_{+}^{5 / 2},
$$

where $\gamma$ is the nonlinear stiffness of Hertzian interactions and $k$ the linear stiffness of local potentials.

Let us first consider two interacting beads, one being initially at rest and the other having an initial velocity $V$, and temporarily neglect the local restoring force of the on-site potentials. After collision, their contact time is approximately equal to $2.43 \tau_{h}$ with $\tau_{h}=\left[m^{2} /\left(\gamma^{2} V\right)\right]^{1 / 5}$, and their maximal compression distance is close to $0.76 \delta$, where $\delta=\left(m V^{2} / \gamma\right)^{2 / 5}[49,21]$. Moreover, the stiffness constant of Hertzian interactions linearized at precompression $\delta$ is of the order of $\kappa_{h}=\gamma \sqrt{\delta}$.

Including back the local restoring forces, the displacement $\xi$ at which Hertzian and local forces equilibrate satisfies $\gamma \xi^{3 / 2}=k \xi$ and is given by $\xi=(k / \gamma)^{2}$. In addition, the period of local oscillations is $2 \pi \tau_{c}$ with $\tau_{c}=(m / k)^{1 / 2}$.

Now we are ready to perform a suitable rescaling of (27). Setting $x_{n}(t)=$ $\xi y_{n}\left(t / \tau_{c}\right)$, the Hamiltonian (27) yields the equations of motion (9) in dimensionless form. Moreover, the initial condition

$$
x_{n}(0)=0, \quad \dot{x}_{n}(0)=V \delta_{n, 1}
$$



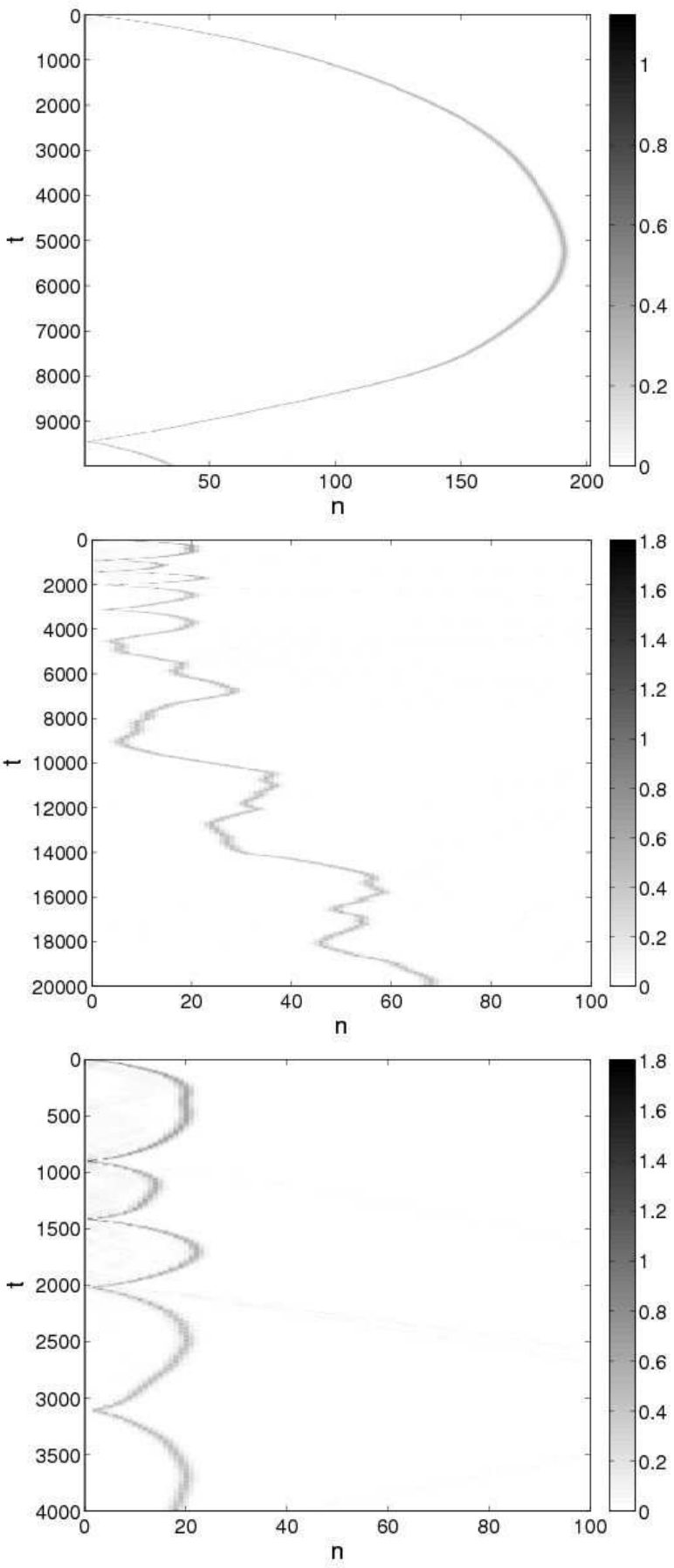

Fig. 22. Space-time diagram giving the energy density (23) after an initial condition of the form (26), for the on-site potential (10) with $s=1$. The upper plot corresponds to $\dot{y}_{1}(0)=1.5$ and the middle plot to $\dot{y}_{1}(0)=1.9$. The bottom plot provides a zoom of the middle one.

reads in dimensionless form

$$
y_{n}(0)=0, \quad \dot{y}_{n}(0)=\lambda^{5 / 2} \delta_{n, 1},
$$



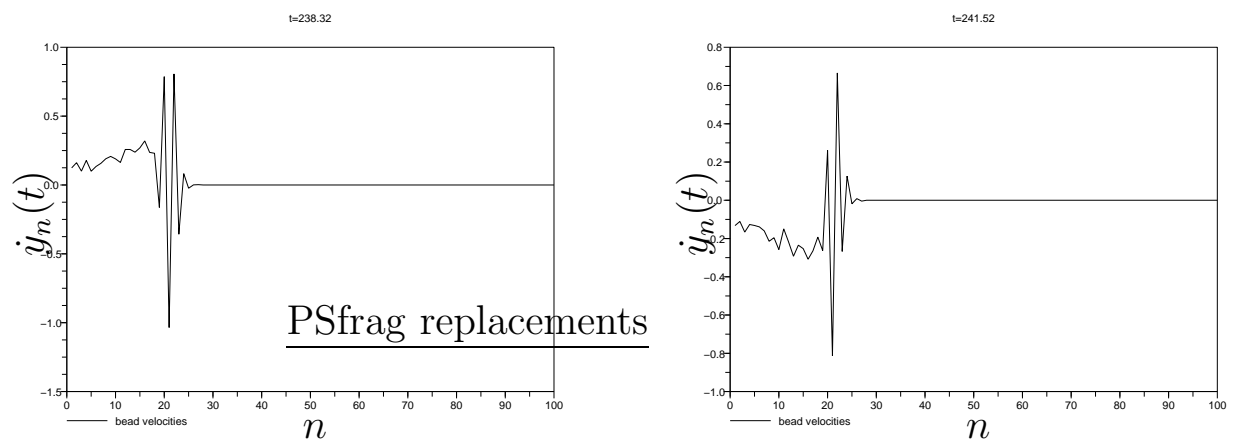

Fig. 23. Particle velocities for $s=1$ and the initial condition $(26)$ with $\dot{y}_{1}(0) \approx 1.87$. The traveling breather profile is shown at two different times close to direction-reversing (the left panel corresponds to $t \approx 238$, and the right panel to $t \approx 241$ ). Nonlinear waves confined between the traveling breather and the boundary are also visible.

where

$$
\lambda=\kappa_{h} / k
$$

measures the relative strengths of the Hertzian interaction at initial velocity $V$ and the local potential. Since $\kappa_{h}=m / \tau_{h}^{2}$, we have equivalently

$$
\lambda=\frac{m}{k \tau_{h}^{2}}
$$

i.e. $\lambda^{1 / 2}$ measures the relative duration of local oscillations and binary collisions.

From (29) we deduce that the DpS regime takes place when $\lambda^{5 / 2}$ is small. For example, for a Newton's cradle with strings of length $50 \mathrm{~cm}$ and binary collision time $2.43 \tau_{h}=0.077 \mathrm{~ms}$ (value taken from [49] for an impact velocity of $\left.1.1 \mathrm{~m} . \mathrm{s}^{-1}\right)$, one obtains $\lambda^{5 / 2} \approx 1.75 \times 10^{19}$, hence we are extremely far from the $\mathrm{DpS}$ regime. In section 3.6 we will introduce a mechanical system for which local oscillators are much stiffer and the DpS dynamics is relevant.

\subsection{A lattice model for cantilevers decorated by spherical beads}

Several types of mechanical models have been devised to analyze the properties of discrete breathers experimentally, see e.g. $[13,63,46,30]$. In this section we introduce a simplified model of the cantilever system sketched in figure 1 (right picture). We consider the form (9) analyzed previously, but examine the more general situation when the lattice is spatially inhomogeneous. With this model, we shall observe that a moving breather generated by an impact on the first cantilever can be almost totally reflected by a localized impurity corresponding to a moderate increase of the bead radii on a single cantilever. 
We begin by introducing a simplified model of the cantilever system of the right panel of) figure 1, where cantilever compression is neglected and bead deformations are treated quasi-statically. More precisely, each bead is seen as an elastic medium at equilibrium, clamped at a cantilever at one side, and either free or in contact with one bead of a neighboring cantilever at the opposite side. So any bead deformation is fully determined by two cantilever positions, and can be approximated by Hertz's contact law. In addition, each cantilever decorated by two spherical beads is described by a point-mass model which approximates the dynamics of the slower bending mode, following a classical approach in the context of atomic force microscope cantilevers [61]. Under these approximations, our model incorporates a single degree of freedom per cantilever, namely its maximal deflection.

The point-mass model is obtained as follows. Using a rod model and under the assumption of small deflection, a cantilever clamped at both ends and bent by a force applied to its mid-point can be represented by an equivalent linear spring of stiffness $k=192 E I \ell^{-3}$, where $E$ is the cantilever's Young modulus, $\ell$ its length and $I=w h^{3} / 12$ its area moment of inertia, $w, h$ denoting the cantilever width and thickness respectively (see e.g. [48], pp. 77 and 81). For a cantilever without attached beads, the first bending mode frequency satisfies $\omega_{\min } \approx$ $22.4[E I /(\rho A)]^{1 / 2} \ell^{-2}([48]$, p.102) where $\rho$ denotes the cantilever density and $A=w h$ its cross section. A single cantilever is then represented by an effective mass $m^{*}=k / \omega_{\min }^{2} \approx 0.38 m_{c}$, where $m_{c}=\rho A \ell$ is the exact cantilever mass. The effective mass of a cantilever decorated by two beads of masses $m_{b}$ is then $m=m^{*}+2 m_{b}$. For beads of radius $R$ and density $d$ we fix consequently $m=0.38 m_{c}+(8 / 3) \pi d R^{3}$.

Now let us describe the model for a one-dimensinal chain of such cantilevers, where all beads are made of the same material with Young's modulus $\mathcal{E}$ and Poisson coefficient $\nu$. We denote by $R_{n}=R \tilde{R}_{n}$ the radius of the two beads of the $n$th cantilever ( $R$ being a reference value and $\tilde{R}_{n}$ an adimensional number), $x_{n}(t)$ the maximal cantilever deflections and $m_{n}=0.38 m_{c}+(8 / 3) \pi d R_{n}^{3}$ their effective masses. The array of decorated cantilevers is then described by the Hamiltonian

$$
\mathcal{H}=\sum_{n} \frac{m_{n}}{2} \dot{x}_{n}^{2}+\frac{k}{2} x_{n}^{2}+\frac{2}{5} \gamma_{n}\left(x_{n}-x_{n+1}\right)_{+}^{5 / 2}
$$

where $\gamma_{n}=\gamma \eta_{n}$ is the nonlinear stiffness constant of Hertzian interactions between two beads on different cantilevers $n$ and $n+1$, defined by $\gamma=\frac{E \sqrt{2 R}}{3\left(1-\nu^{2}\right)}$ and $\eta_{n}=\left[2 \tilde{R}_{n} \tilde{R}_{n+1} /\left(\tilde{R}_{n}+\tilde{R}_{n+1}\right)\right]^{1 / 2}$ (see e.g. [48]).

Setting $x_{n}(t)=\xi y_{n}\left(t / \tau_{c}\right)$ as in section 3.5, the Hamiltonian (31) yields the following equations of motion in dimensionless form

$$
\mu_{n} \ddot{y}_{n}+y_{n}=-\eta_{n}\left(y_{n}-y_{n+1}\right)_{+}^{3 / 2}+\eta_{n-1}\left(y_{n-1}-y_{n}\right)_{+}^{3 / 2},
$$


where $\mu_{n}=m_{n} / m$. Note that if all beads have radius $R$ (i.e. $\tilde{R}_{n}=1$ ) then $\eta_{n}=\mu_{n}=1$.

Our main purpose is to analyze an impact problem in a chain of $N$ cantilevers with free end boundary conditions, where the first cantilever is hit by a striker at $t=0$. For this purpose we consider a simpler initial condition where all cantilevers with index $n \geq 2$ are initially at rest and the first cantilever has initial velocity $V$ and zero deflection. This corresponds to fixing the initial condition (28), which yields (29) in rescaled form.

Numerical simulations are performed for a chain of $N=200$ stainless steel cantilevers with $\rho=8 \times 10^{3} \mathrm{~kg} \cdot \mathrm{m}^{-3}, E=193 \mathrm{GPa}, \ell=25 \mathrm{~mm}, w=5 \mathrm{~mm}$, $h=1 \mathrm{~mm}$, decorated by teflon beads with $d=2.2 \times 10^{3} \mathrm{~kg} \cdot \mathrm{m}^{-3}, \mathcal{E}=1.46 \mathrm{GPa}$, $\nu=0.46$ [60]. All beads have radius $R=2.38 \mathrm{~mm}$, except at the middle of the chain where $\tilde{R}_{100}$ can be tuned. These values correspond to a cantilever array at the macroscopic scale (as in reference [46]), but extensions to the microscale might be also considered $[63,73]$.

We fix the impact velocity $V=1 \mathrm{~m} . \mathrm{s}^{-1}$, which yields $\tau_{h} \approx 0.047 \mathrm{~ms}$. Since $\tau_{c} \approx 0.025 \mathrm{~ms}$, we have $\lambda \approx 0.29$ and $\lambda^{5 / 2}$ is small. Consequently, under the above conditions the DpS approximation is valid in the spatially homogeneous case, or in sufficiently long homogeneous segments of a chain including defects.

The initial impact generates a traveling breather and a fairly extended wavetrain emitted from the boundary, as previously analyzed in section 3.4. The traveling breather velocity is close to 2030 sites per second. Evaluating the traveling breather characteristics at $n=80$, we find a maximal bead velocity close to $0.5 \mathrm{~m} . \mathrm{s}^{-1}$ (i.e. half the impact velocity), a maximal cantilever deflection close to $11 \mu \mathrm{m}$ and a maximal interaction force close to $2.8 \mathrm{~N}$. The pulse duration is close to $3.8 \mathrm{~ms}$ and the period of internal oscillations close to $0.14 \mathrm{~ms} \approx \mathrm{T}_{0} /(1.1), T_{0}=2 \pi \tau_{c}$ being the period of linear local oscillations.

When the breather reaches the defect site, it appears to be almost totally reflected for a large enough inhomogeneity, whereas it remains significantly transmitted for a sufficiently small inhomogeneity. This phenomenon is illustrated by figure 24, which compares the cases $\tilde{R}_{100}=1.6$ (almost total reflection) and $\tilde{R}_{100}=1.1$ (partial reflection). After the breather reflection by the defect for $\tilde{R}_{100}=1.6$, a small part of the vibrational energy remains loosely trapped near the defect site. Such phenomena resulting from breatherdefect interactions have been already numerically observed in different types of Klein-Gordon lattices $[25,72,11]$. In the present model, almost total reflection occurs for physically realistic parameter values, which suggests potential applications of such systems as shock wave reflectors. 

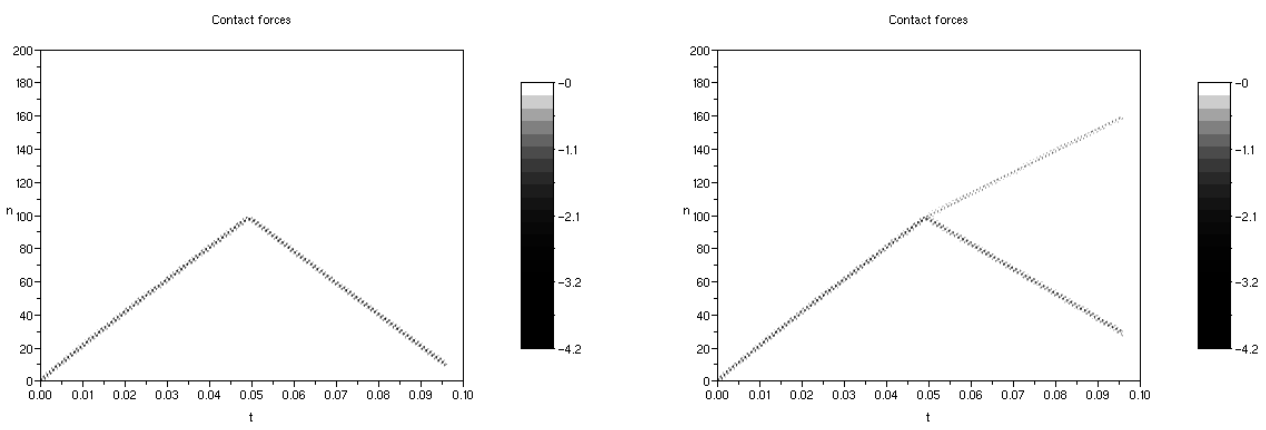

Fig. 24. Space-time diagrams showing the interaction forces $f_{n}=-\gamma_{n}\left(x_{n}-x_{n+1}\right)_{+}^{3 / 2}$ in system (31) for the impact problem described in the text (forces are expressed in $N$ ). Forces are represented in grey levels, white corresponding to vanishing interactions (i.e. beads not in contact) and black to a minimal negative value of the contact force. Left plot : $\tilde{R}_{100}=1.6$. Right plot : $\tilde{R}_{100}=1.1$.

\section{Traveling breathers under precompression}

In section 3 we have analyzed the properties of discrete breathers in chains of oscillators coupled by fully nonlinear Hertzian interactions. We have obtained highly-localized static breathers, which display a super-exponential spatial decay and have an almost constant width in the small amplitude limit. Moreover, small perturbations of the static breathers along a pinning mode generate traveling breathers propagating at an almost constant velocity with very small dispersion.

These properties are largely due to the fully-nonlinear coupling between oscillators, which reduces the phonon band to a single frequency. Intuitively, the absence of linear coupling terms enhances localization, because linear dispersion tends to disperse localized wave packets. Though this phenomenon can be compensated by nonlinearity, breathers in nonlinear lattices with phonon bands generally have a slow exponential spatial decay in the limit of vanishing amplitude (see e.g. [40,38]). Moreover, due to resonance with phonons, exact traveling breathers are generally superposed on nondecaying oscillatory tails, a phenomenon mathematically analyzed in $[36,39,66,37,5]$ (see also section 4.5 of [24] for more references); only under special choices of the speed (or the system parameters) can it then be the case that the amplitude of these oscillatory tails exactly vanishes $[55,56]$.

Due to these noticeably different breather properties in the presence or absence of phonon band, it is interesting to consider physical systems possessing a tunable phonon band, allowing to pass from one situation to the other. This is the case in particular for granular crystals under tunable precompression, since the latter results in a perturbation of the interaction potential inducing an additional harmonic component. In this section, we incorporate this effect 
to model (6), formally analyze the existence of discrete breathers through the phenomenon of modulational instability, and numerically demonstrate that the existence of a phonon band can drastically modify the outcome of an initial impact.

4.1 Granular chain under precompression, and correspondence to the NLS equation

We consider the system (6) with the modified interaction potential

$$
V(r)=\frac{2}{5}(d-r)_{+}^{5 / 2}+d^{3 / 2} r-\frac{2}{5} d^{5 / 2}
$$

where $d>0$ is a parameter. We have thus for $r \approx 0$

$$
V(r)=v_{1} \frac{r^{2}}{2}+v_{2} \frac{r^{3}}{3}+v_{3} \frac{r^{4}}{4}+O\left(|r|^{5}\right)
$$

with

$$
v_{1}=\frac{3}{2} d^{1 / 2}, \quad v_{2}=-\frac{3}{8} d^{-1 / 2}, \quad v_{3}=-\frac{1}{16} d^{-3 / 2} .
$$

This modified potential possesses a harmonic component of size $d^{1 / 2}$ in the neighborhood of the origin, and it becomes linear for $r \geq d$. The first term of (33) corresponds to the classical Hertzian potential including a precompression effect. For example, this type of interaction can be achieved in the cantilever system of figure 1 in the case of a sufficiently long chain. This can be done by applying a force at both ends of the system when the cantilevers are unclamped, which results in a compression of all the beads by a distance $d$ (compression becomes uniform for an infinite system), and by clamping the cantilevers at this new equilibrium state. The second and third terms of (33) do not modify the equations of motion, and just aim at putting the modified Hertz potential in a standard form with $V(0)=0, V^{\prime}(0)=0$.

System (6)-(10)-(33) consists of a mixed Klein-Gordon - FPU lattice, which admits a phonon band with a finite width (of order $O\left(d^{1 / 2}\right)$ when $d \approx 0$ ). The phonons $y_{n}(t)=A e^{i(q n-\omega t)}+$ c.c. of the system linearized at $y_{n}=0$ obey the dispersion relation

$$
\omega^{2}(q)=1+2 v_{1}(1-\cos q),
$$

where $q \in[0, \pi]$ denotes the wavenumber and $\omega$ the phonon frequency.

For this class of systems combining anharmonic local and interaction potentials, the modulational instability (MI) of small amplitude periodic and standing waves has been studied in a number of references $[22,17,43,28,29]$. This phenomenon has been analyzed in $[28,29]$ through the continuum nonlinear 
Schrödinger (NLS) equation, which describes the slow spatio-temporal modulation of small amplitude phonons under the effects of nonlinearity and dispersion (see also the basic papers $[62,47]$ ). From the general results of $[28,29]$, system (6)-(10)-(33) admits solutions of the form

$$
y_{n}(t)=\epsilon A\left[\epsilon^{2} t, \epsilon(n-c t)\right] e^{i(q n-\omega t)}+c . c .+O\left(\epsilon^{3 / 2}\right)
$$

on time intervals of length $O\left(\epsilon^{-2}\right)$ ( $\epsilon$ being a small parameter), where $A(\tau, \xi)$ satisfies the NLS equation

$$
i \partial_{\tau} A=-\frac{1}{2} \omega^{\prime \prime}(q) \partial_{\xi}^{2} A+h|A|^{2} A .
$$

In the above expressions, $\omega$ is given by $(34), c=\omega^{\prime}(q)$ is the group velocity, $h=\beta / \omega, \omega^{\prime \prime}=v_{1} \omega_{2} / \omega$ and

$$
\begin{gathered}
\beta=\frac{16 v_{2}^{2}(\sin q)^{2}(1-\cos q)^{2}}{4 v_{1}(1-\cos q)^{2}+3}+\frac{3}{2}\left[4 v_{3}(1-\cos q)^{2}+s\right], \\
\omega_{2}=\cos q-\frac{v_{1}}{\omega^{2}}(\sin q)^{2}
\end{gathered}
$$

(see [28], equation (2.12) p. 557).

The so-called focusing case of the NLS equation occurs for $\omega^{\prime \prime}(q) h<0$, i.e. under the condition

$$
\Phi:=-\beta \omega_{2}>0 .
$$

In that case the spatially homogeneous solutions of (36) are unstable, and (36) admits sech-shaped solutions corresponding (at least on long transients) to small amplitude traveling breather solutions taking the form

$$
y_{n}(t)=\epsilon M \frac{e^{i\left[q n-\left(\omega-\epsilon^{2} \omega^{\prime \prime} / 2\right) t+\varphi\right]}}{\cosh [\epsilon(n-c t)]}+c . c .+O\left(\epsilon^{3 / 2}\right),
$$

where $M=\left(-\omega^{\prime \prime} / h\right)^{1 / 2}$. These solutions decay exponentially in space and broaden in the small amplitude limit $\epsilon \rightarrow 0$, in contrast with the traveling breathers numerically obtained in section 3.4 in the absence of precompression. For Klein-Gordon lattices (i.e. for harmonic interaction potentials), the existence of exact traveling breather solutions close to (38) has been proved in special cases in $[36,39,66]$ (breathers are superposed on a nondecaying oscillatory tail, exponentially small in $\epsilon$ ). However these results do not directly apply to our model having anharmonic interaction potentials.

The frequency $\omega(q)$ defined by (34) admits a unique inflection point in the interval $(0, \pi)$, at the wavenumber $q=q_{c} \in(0, \pi / 2)$ satisfying $\cos q_{c}=v_{1}(1-$ $\left.\cos q_{c}\right)^{2}$. In the generic case when $\beta\left(q_{c}\right) \neq 0$, it follows that $\Phi$ changes sign at $q=q_{c}$ (since $w_{2}$ changes sign). Consequently, MI generically occurs for wavenumbers in some interval lying at one side of $q_{c}$. This interval may extend 
or not up to one edge of the phonon band, depending on parameter values. For $q=0$ (in-phase mode) the condition $\Phi>0$ reduces to $s<0$, and for $q=\pi$ (out-of-phase mode) it reduces to $16 v_{3}+s>0$. These conditions have been also obtained in [22] through a Hill's type analysis.

In the next section, we numerically check that MI can lead to the formation of traveling breathers on long transients, and revisit the impact problem of section 3.4 .

\subsection{Excitation of traveling breathers}

In this section we fix $d=1 / 2$, so that $v_{1} \approx 1.06, v_{2} \approx-0.53$ and $v_{3} \approx-0.17$, and consider different values of the anharmonicity parameter $s=1, s=0$ and $s=-1 / 6$. For all these values, there exists a band of unstable phonon modes characterized by $\Phi(q)>0$ (see figure 25). One can notice that $\Phi$ is much smaller for $s=0$ inside the band of unstable modes, due to the smallness of $h$. In that case, slower MI should occur according to the NLS approximation, but at the same time the applicability of the latter should be restricted to smaller values of $\epsilon$.

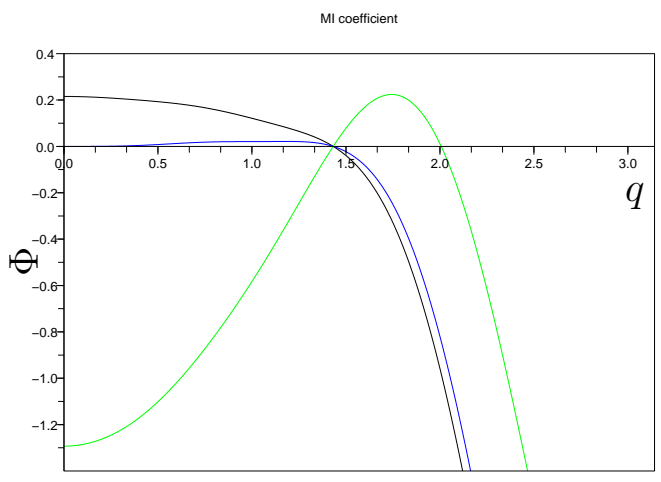

Fig. 25. Graphs of the MI coefficient $\Phi$ defined by (37) as a function of wavenumber $q$, for $s=-1 / 6$ (black curve), $s=0$ (blue curve), $s=1$ (green curve). Modulational instability occurs in the bands where $\Phi>0$.

To illustrate the MI phenomenon, we integrate (6)-(10)-(33) numerically for initial conditions

$$
\begin{aligned}
& x_{n}(0)=a \sin (q n)(1+b \cos (2 n \pi / N)), \\
& \dot{x}_{n}(0)=-a \omega \cos (q n)(1+b \cos (2 n \pi / N))
\end{aligned}
$$

corresponding to slowly modulated phonons, with $a=0.15, b=0.01$, a wavenumber $q$ in the band of unstable modes (see fig. 25), and $\omega$ determined 

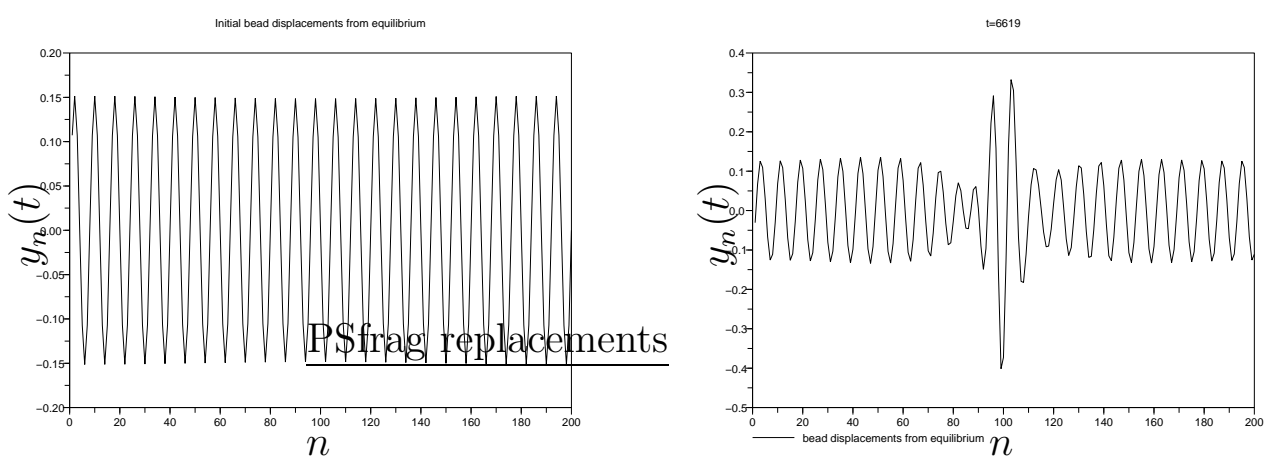

Fig. 26. Evolution of particle positions in the system (6)-(10)-(33) with periodic boundary conditions $(N=200$ particles). We consider the case $s=-1 / 6$ with precompression $d=0.5$. The initial condition (39) is plotted in the left panel (case $q=\pi / 4$ ). Particle displacements plotted at time $t=6619$ (right panel) reveal the formation of a traveling breather resulting from a modulational instability (the envelope propagates rightwise).

by (34). We consider a chain of $N$ particles with periodic boundary conditions. Figure 26 displays the results for $s=-1 / 6, q=\pi / 4$ and $N=200$. The initial perturbation generates a traveling breather over a long transient (at the end of which a splitting of the pulse occurs). The same phenomenon occurs for $s=1$ and $s=0$, albeit the latter case results in slower instabilities and less localized traveling breathers (results not shown).

According to the previous computations, traveling breathers with profiles reminiscent of (38) can be generated from slow modulations of small amplitude unstable phonons. This raises the question of the nucleation of traveling breather from other types of initial conditions, in particular for a localized impact. In section 3.4 we observed that this type of excitation systematically generates traveling breathers. By extending this study to the case of potential (33), we will show in which way linear dispersion may modify the impact dynamics.

In what follows we keep the same values of parameters $d, s$ and integrate (6)(10)-(33) numerically (for free end boundary conditions), starting from the initial condition $(26)$ with $\dot{y}_{1}(0) \approx 1.87$. Depending on the value of $s$, the initial excitation may lead to different dynamical phenomena, and notable differences with respect to the case without precompression are observed.

The case $s=1$ is described in figure 27, which shows the particle velocity profiles at different times. A traveling breather reminiscent of the sech-type envelope solitons (38) appears after the impact. It forms around $t=290$ (top plot), and remains much less localized than the traveling breathers previously obtained without precompression (compare figures 27 and 23). The breather propagates away from the boundary (middle and bottom plots) and the "boomerang effect" that occurs without precompression disappears. In addition, the initial perturbation generates a dispersive wavetrain of substantial 
amplitude, and the traveling breather becomes ultimately superposed on an oscillatory tail at both sides of the central pulse (bottom plot), which yields a traveling breather profile reminiscent of the waves computed in [67] (see also [56]).
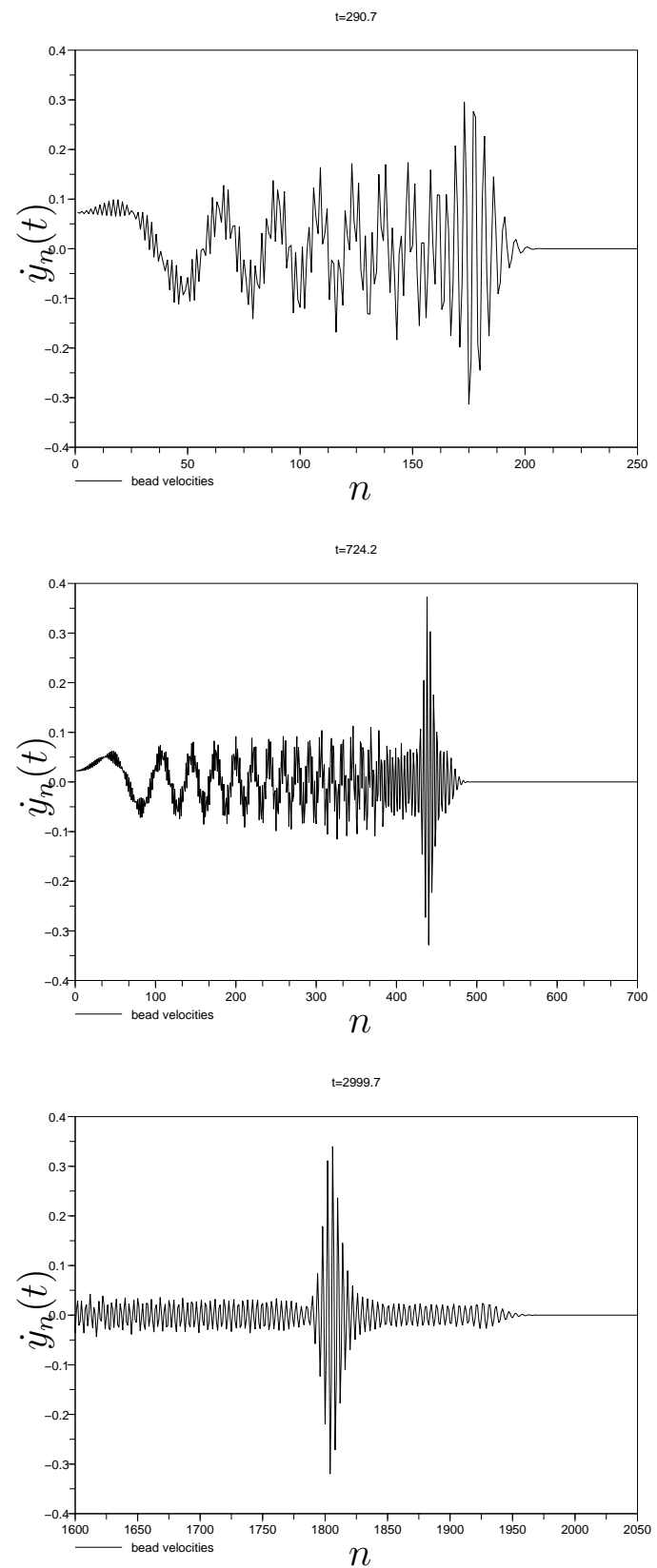

Fig. 27. Snapshot of particle velocities in system (9)-(10)-(33) with anharmonicity parameter $s=1$ and precompression $d=0.5$, for the initial condition (26) with $\dot{y}_{1}(0) \approx 1.87$. The profile is plotted at three different times $t \approx 291$ (top panel), $t \approx 724$ (middle panel) and $t \approx 3000$ (bottom panel), showing the formation of a traveling breather that coexists with a sizeable dispersive wavetrain. 
The cases $s=-1 / 6$ and $s=0$ yield a different situation described in figure 28. The difference with the case without precompression is striking (compare figure 28 with figure 21). The initial localized perturbation generates an important dispersive wavetrain, and no traveling breather is excited, at least on the timescales of the simulation. Moreover, in the present case we do not observe the formation of a surface mode.

As a conclusion, according to our results, introducing a precompression attenuates spatial localization and enhances dispersive effects, a phenomenon linked with an additional linear component embedded within the Hertzian interactions.
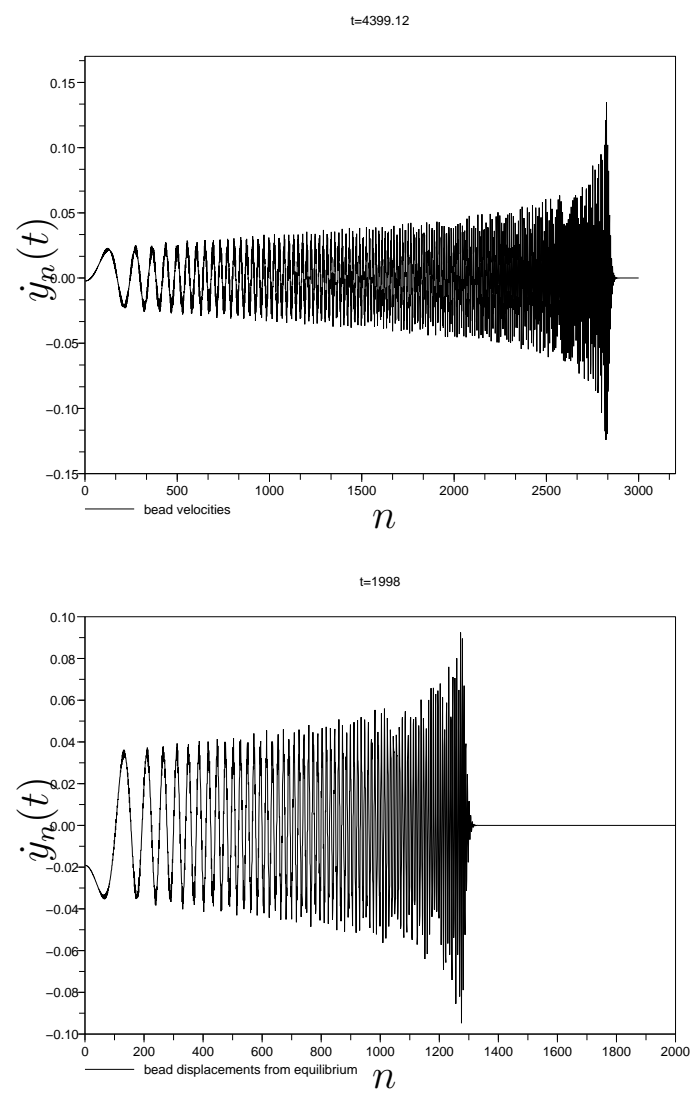

Fig. 28. Same as in figure 27 , for $s=0$ (top panel, particle velocities at $t \approx 4399$ ) and $s=-1 / 6$ (bottom panel, particle velocities at $t \approx 1998$ ).

\section{Conclusion}

We have analyzed the properties of discrete breathers in FPU lattices and mixed FPU-Klein-Gordon lattices with Hertzian interactions. While static breathers don't exist in the absence of precompression and of onsite potentials, 
the addition of the latter creates highly localized breathers, which display a particularly strong mobility, a phenomenon well-described by the DpS equation in the small amplitude regime and associated with the spectral properties (i.e., the pinning mode) of such states. Beyond the DpS limit, we have identified different phenomena depending on the softening or hardening character of the local potential, namely the generation of a surface mode after an impact or the existence of direction-reversing traveling breathers. Importantly also the stability of both the on-site and inter-site breather states obtained was critically dependent on the strength (and sign) of the anharmonicity.

We have also introduced a mechanical model consisting of a chain of stiff cantilevers decorated by spherical beads, which may allow to realize the above localized excitations. According to our study, an impact at one end of the cantilever chain should generate a highly-localized traveling breather. In this regime, contrary to what is the case for a regular cradle under gravity, the ranges of parameters of the problem (e.g., beads of about $1 \mathrm{~cm}$ diameter, loads of about $1 \mathrm{~N}$, and cantilever width of about $1 \mathrm{~cm}$ ) are deemed relevant for the observation of such breathers and for the description of the system by the DpS approximation examined herein.

Obviously, one has to stress that the lattice model (32) is simplified and important corrections may apply. For example, a finite-element modeling would be helpful to validate the model or improve its calibration. In addition, it would be important to take dissipation into account, following e.g. the approach of [8]. Since many sources of dissipation are present (friction, plasticity effects, transmission of vibrational energy through the walls), one can wonder if dissipation may overdamp the dynamics and completely destroy the breathers. However, recent experimental results [7] have demonstated that static breathers with lifetimes of the order of $10 \mathrm{~ms}$ could be generated in diatomic granular chains. During this time, the moving breather computed in section 3.6 would travel over approximately 20 sites (performing roughly 70 internal oscillations), which would allow for an experimental detection, provided this excitation persists in the presence of dissipation, with moderate changes in velocity and frequency. Although the setting of decorated cantilevers proposed herein would have the additional source of dissipation through radiating energy into the ground (through the clamping of the cantilevers), it is certainly deemed worthwhile to consider such experimental setups and to examine systematically the resulting dynamics.

A different approach which may allow to generate static breathers is linked with modulational instability. Indeed, static breathers have been excited by modulational instabilities in experiments performed on diatomic granular chains [7], a phenomenon also numerically illustrated in the Newton's cradle [41]. In this respect, an extensive study of MI in the cradle model (with the help of the DpS equation) would be of interest. A related aspect concerns the actuation 
of the system through the driving of a bead with a particular frequency. In fact, the experiments of [7] were realized based on such actuation of the chain at modulationally unstable frequencies rather than the generation of suitable spatially extended, modulationally unstable states. In that regard, it should be noted that it is not straightforward to experimentally initialize desired spatial profiles throughout the lattice in this system.

As we have seen, static breathers may be deformed by weak instabilities resulting in a translational motion and traveling counterparts thereof. However, in an experimental context, these weak instabilities are likely to be irrelevant due to dissipation. To fix the ideas, let us assume a breather lifetime of the order of $10 \mathrm{~ms}$ in the presence of dissipation, as in the experiments of [7]. In the computations of section 3.6, the breather periods at small amplitude were (roughly) close to $0.15 \mathrm{~ms}$, therefore unstable Floquet eigenvalues $1+\epsilon$ would have an effect over times of order $0.15 \epsilon^{-1} \mathrm{~ms}$. Consequently, dissipation should destroy the breather well before the instability becomes observable as soon as $\epsilon<0.015$, and thus the instabilities identified in section 3.3 (where $\epsilon<10^{-3}$ ) would be largely dominated by dissipative effects.

From a numerical point of view, an interesting open problem concerns the computation of traveling breathers. In the above computations, approximate traveling breathers were generated by the dynamics after an impact at one end of the chain. It would be interesting to compute exact traveling breather solutions using the Newton method, as in references $[2,67,56]$. Moreover, the existence (and physical explanation) of direction-reversing traveling breathers remains to be elucidated. Furthermore, it would be relevant to understand in more detail the nature of interactions of these traveling breather with static defects. Studies in these directions are currently in progress and will be reported in future publications.

Acknowledgements. Part of this work has been carried out during a visit of P.G.K. to laboratoire Jean Kuntzmann, supported by the CNRS, France, to which P.G.K. is grateful for the hospitality. The authors are grateful to Nicholas Boechler for helpful inputs on experimental issues. G.J. acknowledges stimulating discussions with E. Dumas, B. Bidegaray, M. Peyrard and G. Theocharis. J.C. acknowledges financial support from the MICINN project FIS2008-04848. P.G.K. also acknowledges support from the US National Science Foundation through grant CMMI-1000337 and also from the Alexander S. Onassis Public Benefit Foundation through the grant RZG 003/2010-2011. 


\section{References}

[1] K. Ahnert and A. Pikovsky. Compactons and chaos in strongly nonlinear lattices, Phys. Rev. E 79 (2009), 026209.

[2] S. Aubry and T. Cretegny. Mobility and reactivity of discrete breathers, Physica D 119 (1998), 34-46.

[3] S. Aubry, G. Kopidakis and V. Kadelburg. Variational proof for hard discrete breathers in some classes of Hamiltonian dynamical systems, Discrete Contin. Dyn. Syst. Ser. B 1 (2001), 271-298.

[4] S. Aubry. Discrete breathers : localization and transfer of energy in discrete Hamiltonian nonlinear systems, Physica D 216 (2006), 1-30.

[5] O.F. Oxtoby, I.V. Barashenkov, Moving solitons in the discrete nonlinear Schrödinger equation, Phys. Rev. E 76 (2007), 036603.

[6] N. Boechler, G. Theocharis and C. Daraio. Bifurcation-based acoustic switching and rectification, Nat. Mater. (2011), DOI:10.1038/NMAT3072.

[7] N. Boechler, G. Theocharis, S. Job, P.G. Kevrekidis, M.A. Porter and C. Daraio. Discrete breathers in one-dimensional diatomic granular crystals, Phys. Rev. Lett. 104 (2010), 244302.

[8] R. Carretero-González, D. Khatri, M.A. Porter, P.G. Kevrekidis and C. Daraio. Dissipative solitary waves in granular crystals, Phys. Rev. Lett. 102 (2009), 024102 .

[9] D. Chen, S. Aubry and G.P. Tsironis. Breather mobility in discrete $\phi^{4}$ lattices, Phys. Rev. Lett. 77 (1996), 4776.

[10] T. Cretegny and S. Aubry. Spatially inhomogeneous time-periodic propagating waves in anharmonic systems, Phys. Rev. B 55 (1997), R11 929-932.

[11] J. Cuevas, F. Palmero, J.F.R. Archilla and F.R. Romero. Moving discrete breathers in a Klein-Gordon chain with an impurity, J. Phys. A: Math. Gen. 35 (2002), 10519-10530.

[12] J. Cuevas, G. James, P.G. Kevrekidis, B. Malomed and B. Sánchez-Rey, Approximation of solitons in the discrete NLS equation, Journal of Nonlinear Mathematical Physics 15, supplement 3 (2008), 124-136.

[13] J. Cuevas, L.Q. English, P.G. Kevrekidis and M. Anderson. Discrete breathers in a forced-damped array of coupled pendula: modeling, computation, and experiment, Phys. Rev. Lett. 102 (2009), 224101.

[14] T. Dauxois, M. Peyrard and C.R. Willis. Discreteness effects on the formation and propagation of breathers in nonlinear Klein-Gordon equations, Phys. Rev. E 48 (1993), 4768. 
[15] Q. Dou, J. Cuevas, J.C. Eilbeck and F.M. Russell. Breathers and kinks in a simulated breather experiment, Discrete Contin. Dyn. Syst. Ser. S 4 (2011), 1107-1118.

[16] A. Degasperis, M. Conforti, F. Baronio and S. Wabnitz. Effects of nonlinear wave coupling: Accelerated solitons, Eur. Phys. J. Special Topics 147 (2007), 233-252.

[17] J. Dorignac and S. Flach. Isochronism and tangent bifurcation of band edge modes in Hamiltonian lattices, Physica D 204 (2005), 83-109.

[18] E. Dumas. Personal communication. 2011.

[19] J.C. Eilbeck and M. Johansson. The discrete nonlinear Schrödinger equation 20 years on. In : L. Vazquez, R.S. MacKay, M-P. Zorzano, editors, Conference on Localization and Energy Transfer in Nonlinear Systems, p.44, World Scientific (2003).

[20] J.M. English and R.L. Pego. On the solitary wave pulse in a chain of beads, Proc. Amer. Math. Soc. 133, n. 6 (2005), 1763-1768.

[21] E. Falcon. Comportements dynamiques associés au contact de Hertz : processus collectifs de collision et propagation d'ondes solitaires dans les milieux granulaires, PhD thesis, Université Claude Bernard Lyon 1 (1997).

[22] S. Flach. Tangent bifurcation of band edge plane waves, dynamical symmetry breaking and vibrational localization, Physica D 91 (1996), 223-243.

[23] S. Flach and C.R. Willis. Discrete Breathers, Physics Reports 295 (1998), 181264.

[24] S. Flach and A. Gorbach. Discrete breathers : advances in theory and applications, Physics Reports 467 (2008), 1-116.

[25] K. Forinash, M. Peyrard and B. Malomed. Interaction of discrete breathers with impurity modes, Phys Rev E 49 (1994), 3400-3411.

[26] F. Fraternali, M. A. Porter, and C. Daraio. Optimal design of composite granular protectors, Mech. Adv. Mat. Struct. 17 (2010), 1-19.

[27] G. Friesecke and R.L. Pego. Solitary waves on FPU lattices : I. Qualitative properties, renormalization and continuum limit, Nonlinearity 12 (1999), 16011627.

[28] J. Giannoulis and A. Mielke. The nonlinear Schrödinger equation as a macroscopic limit for an oscillator chain with cubic nonlinearities, Nonlinearity 17 (2004), 551-565.

[29] J. Giannoulis and A. Mielke. Dispersive evolution of pulses in oscillator chains with general interaction potentials, Discrete Contin. Dyn. Syst. Ser. B 6 (2006), 493-523.

[30] S.C. Green, C.J. Budd and G.W. Hunt. Breathers in a pinned mechanical lattice, SIAM J. Appl. Dyn. Syst. 10 (2011), 66-91. 
[31] E. Hascoët and H.J. Herrmann. Shocks in non-loaded bead chains with impurities, Eur. Phys. J. B 14 (2000), 183-190.

[32] E. J. Hinch and S. Saint-Jean. The fragmentation of a line of ball by an impact, Proc. R. Soc. London, Ser. A 455 (1999), 3201-3220.

[33] J. Hong. Universal power-law decay of the impulse energy in granular protectors, Phys. Rev. Lett. 94 (2005), 108001.

[34] H. Hu, A. Strybulevych, J. H. Page, S. E. Skipetrov and B. A. van Tiggelen. Localization of ultrasound in a three-dimensional elastic network, Nature Physics 4 (2008), 945.

[35] S. Hutzler, G. Delaney, D. Weaire and F. MacLeod. Rocking Newton's cradle, Am. J. Phys. 72, n.12 (2004), 1508-1516.

[36] G. Iooss and K. Kirchgässner. Travelling waves in a chain of coupled nonlinear oscillators, Commun. Math. Phys. 211 (2000), 439-464.

[37] G. Iooss and G. James. Localized waves in nonlinear oscillator chains, Chaos 15 (2005), 015113.

[38] G. James and P. Noble. Breathers on diatomic Fermi-Pasta-Ulam lattices, Physica D 196 (2004), 124-171.

[39] G. James and Y. Sire. Travelling breathers with exponentially small tails in a chain of nonlinear oscillators, Commun. Math. Phys. 257 (2005), 51-85.

[40] G. James, B. Sánchez-Rey and J. Cuevas. Breathers in inhomogeneous lattices : an analysis via center manifold reduction, Reviews in Mathematical Physics 21 (2009), 1-59.

[41] G. James. Nonlinear waves in Newton's cradle and the discrete $p$-Schrödinger equation (2010), arXiv:1008.1153v1 [nlin.PS]. To appear in Math. Mod. Meth. Appl. Sci.

[42] S. Job, F. Santibanez, F. Tapia and F. Melo. Wave localization in strongly nonlinear Hertzian chains with mass defect, Phys. Rev. E 80 (2009), 025602(R).

[43] M. Johansson. Discrete nonlinear Schrödinger approximation of a mixed KleinGordon/Fermi-Pasta-Ulam chain : modulational instability and a statistical condition for creation of thermodynamic breathers, Physica D 216 (2006), 6270 .

[44] M. Kastner and J-A. Sepulchre. Effective Hamiltonian for traveling discrete breathers in the FPU chain, Discrete Contin. Dyn. Syst. Ser. B 5 (2005), 719734 .

[45] P.G. Kevrekidis, The Discrete Nonlinear Schrödinger Equation: Mathematical Analysis, Numerical Computations and Physical Perspectives, Springer-Verlag (Heidelberg, 2009). 
[46] M. Kimura and T. Hikihara. Coupled cantilever array with tunable on-site nonlinearity and observation of localized oscillations, Physics Letters A 373 (2009), 1257-1260.

[47] V.V. Konotop. Small-amplitude envelope solitons in nonlinear lattices, Phys. Rev. E 53 (1996), 2843-2858.

[48] L.D. Landau, E.M. Lifshitz, A.M. Kosevich and L.P. Pitaevskii. Theory of Elasticity, Third Edition (Theoretical Physics, Vol 7), Butterworth-Heinemann (1986).

[49] D.R. Lovett, K.M. Moulding and S. Anketell-Jones. Collisions between elastic bodies : Newton's cradle, Eur. J. Phys. 9 (1988), 323-328.

[50] R.S. MacKay and S. Aubry. Proof of existence of breathers for time-reversible or Hamiltonian networks of weakly coupled oscillators, Nonlinearity 7 (1994), 1623-1643.

[51] R.S. MacKay. Solitary waves in a chain of beads under Hertz contact, Phys. Lett. A 251 (1999), 191-192.

[52] R.S. MacKay and J.-A. Sepulchre. Effective Hamiltonian for travelling discrete breathers, J. Phys. A 35 (2002), 3985-4002.

[53] J.L. Marin and S. Aubry. Breathers in nonlinear lattices : numerical calculation from the anticontinuous limit, Nonlinearity 9 (1996), 1501-1528.

[54] J.L. Marin and S. Aubry. Finite size effects on instabilities of discrete breathers, Physica D 119 (1998), 163-174.

[55] T.R.O. Melvin, A.R. Champneys, P.G. Kevrekidis and J. Cuevas, Radiationless traveling waves in saturable nonlinear Schrödinger lattices, Phys. Rev. Lett. 97 (2006), 124101.

[56] T.R.O. Melvin, A.R. Champneys, P.G. Kevrekidis and J. Cuevas, Travelling solitary waves in the discrete Schrödinger equation with saturable nonlinearity: Existence, stability and dynamics, Physica D 237, (2008), 551-567.

[57] M.I. Molina, R.A. Vincencio and Yu.S. Kivshar. Discrete solitons and nonlinear surface modes in semi-infinite waveguide arrays, Optics letters 31 (2006), 16931695.

[58] V.F. Nesterenko, Dynamics of heterogeneous materials, Springer Verlag, 2001.

[59] A. Pankov. Travelling waves and periodic oscillations in Fermi-Pasta-Ulam lattices, Imperial College Press, London, 2005.

[60] M. Porter, C. Daraio, I. Szelengowicz, E.B. Herbold and P.G. Kevrekidis. Highly nonlinear solitary waves in heterogeneous periodic granular media, Physica $D$ 238 (2009), 666-676.

[61] U. Rabe, K. Janser and W. Arnold. Vibrations of free and surface-coupled atomic force microscope cantilevers : Theory and experiments. Rev. Sci. Instrum. 67 (1996), 3281-3293. 
[62] M. Remoissenet. Low-amplitude breather and envelope solitons in quasi-onedimensional physical models, Phys. Rev. B 33 (1986), 2386-2392.

[63] M. Sato, B.E. Hubbard and A.J. Sievers. Nonlinear energy localization and its manipulation in micromechanical oscillator arrays, Review of modern physics 78 (2006), 137-155.

[64] S. Sen, J. Hong, J. Bang, E. Avalos and R. Doney. Solitary waves in the granular chain, Physics Reports 462 (2008), 21-66.

[65] J-A. Sepulchre. Energy barriers in coupled oscillators : from discrete kinks to discrete breathers. In : L. Vazquez, R.S. MacKay, M-P. Zorzano, editors, Conference on Localization and Energy Transfer in Nonlinear Systems, p.102129, World Scientific (2003).

[66] Y. Sire. Travelling breathers in Klein-Gordon lattices as homoclinic orbits to p-tori, J. Dyn. Diff. Eqs. 17 (2005), 779-823.

[67] Y. Sire and G. James. Numerical computation of travelling breathers in KleinGordon chains, Physica D 204 (2005), 15-40.

[68] A. Spadoni and C. Daraio. Generation and control of sound bullets with a nonlinear acoustic lens, Proc Natl Acad Sci USA 107 (2010), 7230.

[69] A. Stefanov and P.G. Kevrekidis, On the existence of solitary traveling waves for generalized Hertzian chains, arXiv:1105.3364.

[70] G. Theocharis, M. Kavousanakis, P.G. Kevrekidis, C. Daraio, M.A. Porter and I.G. Kevrekidis. Localized breathing modes in granular crystals with defects, Phys. Rev. E 80 (2009), 066601.

[71] G. Theocharis, N. Boechler, P. G. Kevrekidis, S. Job, M.A. Porter, and C. Daraio. Intrinsic energy localization through discrete gap breathers in onedimensional diatomic granular crystals, Phys. Rev. E. 82 (2010), 056604.

[72] J.J.L. Ting and M. Peyrard. Effective breather trapping mechanism for DNA transcription, Phys Rev E 53 (1996), 1011-1020.

[73] M.K. Yapici and J. Zou. Microfabrication of colloidal scanning probes with controllable tip radii of curvature, J. Micromech. Microeng. 19 (2009), 105021. 